\title{
Instability of asymptotically anti-de Sitter black holes under Robin conditions at the timelike boundary
}

\author{
Bernardo Araneda* and Gustavo Dotti ${ }^{\dagger}$ \\ Facultad de Matemática, Astronomía y Física, Universidad Nacional de Córdoba, Instituto de Física \\ Enrique Gaviola, Conicet. Ciudad Universitaria, (5000) Córdoba, Argentina \\ (Received 23 February 2017; revised manuscript received 16 August 2017; published 15 November 2017)
}

\begin{abstract}
The static region outside the event horizon of an asymptotically anti-de Sitter black hole has a conformal timelike boundary $\mathcal{I}$ on which boundary conditions have to be imposed for the evolution of linear fields from initial data to be a well-posed problem. Only homogeneous Dirichlet, Neumann or Robin conditions preserve the action of the background isometry group on the solution space. We study the case in which the modal decomposition of the linear field leads to potentials not diverging at the conformal timelike boundary. We prove that there is always an instability if Robin boundary conditions with large enough $\gamma$ (the quotient between the values of the derivative of the field and the field at the boundary) are allowed. We explain the origin of this instability, show that for modes with non-negative potentials there is a single unstable state and prove a number of properties of this state. Although our results apply, in general, to $1+1$ wave equations on a half-infinite domain with a potential that is not singular at the boundary, our motivation is to analyze the gravitational stability of the four-dimensional Schwarzschild anti-de Sitter black holes in the context of the black hole nonmodal linear stability program initiated in Phys. Rev. Lett. 112, 191101 (2014), and the related supersymmetric type of duality exchanging odd and even modes. We prove that this symmetry is broken except when a combination of Dirichlet conditions in the even sector and a particular Robin condition in the odd sector is enforced, or vice versa, and that only the first of these two choices leads to stable dynamics.
\end{abstract}

DOI: 10.1103/PhysRevD.96.104020

\section{INTRODUCTION}

A preliminary stability criterion for a stationary black hole is that linear fields on the outer stationary region do not grow unbounded from initial data. Fields of interest are Klein-Gordon, Maxwell and linear perturbations of the metric. The evolution of linearized metric perturbations is particularly important because it gives a hint about the ultimate question of full nonlinear stability, which is whether or not generic initial data for the gravitational field equations, close to that of the black hole, will evolve into spacetimes that asymptotically approach stationary black holes of similar characteristics. There are cases, however, where the complexity of the metric and the field equations make even an integral treatment of the linear gravity problem particularly complex (some examples are higher dimensional hairy black holes in generalized gravity theories). In those cases the stability of scalar and/or Maxwell fields is often considered as indicative of linear gravity stability.

In any case, the stability notion assumes unique evolution from initial data, which, given that the fields of interest obey hyperbolic equations, is guaranteed only if the outer region is globally hyperbolic. Asymptotically anti-de Sitter spacetimes, however, are not globally hyperbolic; they have

\footnotetext{
*baraneda@famaf.unc.edu.ar

†gdotti@famaf.unc.edu.ar
}

a conformal timelike boundary $\mathcal{I}$ where boundary conditions have to be imposed to guarantee unambiguous evolution from initial data. When different choices of boundary conditions are possible, they lead to different dynamics outside the domain of dependence of the initial data hypersurface, and therefore to potentially different answers to the issue of stability.

We are interested in the four-dimensional Schwarzschild black hole solution of general relativity (GR)

$$
d s^{2}=-f(r) d t^{2}+\frac{d r^{2}}{f(r)}+r^{2}\left(d \theta^{2}+\sin ^{2} \theta d \varphi^{2}\right),
$$

when the cosmological constant $\Lambda$ in

$$
f(r)=1-2 M / r-\Lambda r^{2} / 3,
$$

is chosen to be negative ( $M>0$ is the mass). This is the Schwarzschild anti-de Sitter black hole in four dimensions $\left(\mathrm{SAdS}_{4}\right)$, for which $f$ has a unique positive root at $r=r_{h}$, the horizon radius, in terms of which

$$
f(r)=1-\frac{\left(1-\frac{1}{3} \Lambda r_{h}^{2}\right) r_{h}}{r}-\frac{\Lambda r^{2}}{3} .
$$

In [1] a definite answer to the linear stability problem for the Schwarzschild black hole $[\Lambda=0$ in (2)] was given, by showing that generic metric perturbations will remain 
bounded and that the perturbed metric will approach asymptotically that of a slowly rotating Kerr black hole. All previous works on Schwarzschild linear stability had been restricted to isolated harmonic modes. The proof of nonmodal linear stability in [1] was extended to the $\Lambda>0$ case in [2]. Crucial to this proof is the use of the duality between odd and even Schwarzschild perturbation discovered by Chandrasekhar [3]. This duality gives a bijection between even and odd parity modes [1,2], and this bijection allows us to write even perturbations in terms of solutions of the odd perturbation Regge-Wheeler equation in $(t, r)$ space (for a detailed proof see Lemma 7 in [2]). The even mode Zerilli equation is therefore avoided and the linear stability problem for the $\Lambda \geq 0$ Schwarzschild black hole shown to reduce to the study of a four-dimensional ReggeWheeler equation for a scalar field $\Phi$

$$
\nabla_{\alpha} \nabla^{\alpha} \Phi+\left(\frac{M}{r^{3}}-\frac{2 \Lambda}{3}\right) \Phi=0
$$

on the $\Lambda \geq 0$ background [1,2].

Two difficulties arise when trying to generalize these ideas to $\mathrm{SAdS}_{4}$ :

(a) one, of a fundamental nature, is that there are different dynamics depending on the boundary conditions at the timelike conformal boundary,

(b) the other one is that for most boundary conditions Chandrasekhar's duality, which is instrumental in the treatment of the $\Lambda \geq 0$ cases, is broken.

We introduce, as usual, a "tortoise" coordinate $x$

$$
x=-\int_{r}^{\infty} \frac{d r^{\prime}}{f\left(r^{\prime}\right)} .
$$

If $\Lambda$ were non-negative, we would find that $x \in(-\infty, \infty)$; however, for negative $\Lambda$ we find that $x \in(-\infty, 0)$ with $x \rightarrow$ $-\infty$ as $r \rightarrow r_{h}{ }^{+}$and $x \rightarrow 0$ as $r \rightarrow \infty$ in the following way:

$$
x \simeq \begin{cases}\frac{r_{h}}{1-\Lambda r_{h}{ }^{2}} \ln \left(\frac{r}{r_{h}}-1\right), & r \rightarrow r_{h}^{+} \\ \frac{3}{\Lambda r}, & r \rightarrow \infty .\end{cases}
$$

In terms of $x$ the metric on the static region reads

$$
d s^{2}=f\left(-d t^{2}+d x^{2}\right)+r^{2}\left(d \theta^{2}+\sin ^{2} \theta d \phi^{2}\right),
$$

where $r=r(x)$ is the inverse of (5). This metric is defined on the manifold $\mathbb{R}_{t} \times(-\infty, 0)_{x} \times S^{2}$ and has the same causal structure of $S^{2}$ times the $x<0$ half of Minkowski space in $1+1$ dimensions, which is not a globally hyperbolic spacetime: given any spacelike hypersurface $\Sigma$ there will be causal lines not intersecting it (e.g., those which are future directed and originate at an $x=0$ point to the future of $\Sigma$ ). Fields obeying wavelike equations are no longer determined by their values and time derivatives at, say, a $t=t_{o}$ surface $\Sigma_{t_{o}}$. The differential equation they satisfy has a unique solution only within the domain of dependence of $\Sigma_{t_{o}}$, and this is not the entire space. To assure uniqueness on the entire space, boundary conditions at the conformal timelike boundary $x=0$ have to be specified. Different boundary conditions may be consistent with the field equations and yet lead to different evolutions of the same initial datum.

The situation of $\mathrm{SAdS}_{4}$ generalizes to the large class of asymptotically anti-de Sitter (AdS) static black hole solutions of $d=n+2$ dimensional GR with the horizon an Einstein manifold $\sigma^{n}$ with metric $\hat{g}_{A B}$ and Ricci tensor $\hat{R}_{A B}=(n-1) \kappa \hat{g}_{A B}, \kappa=0, \pm 1$. The metric of these black holes in static coordinates $\left(t, r, z^{A}\right)$ is given by $[4,5]$

$$
d s^{2}=-f(r) d t^{2}+\frac{d r^{2}}{f(r)}+r^{2} \hat{g}_{A B}(z) d z^{A} d z^{B},
$$

with

$$
f(r)=\kappa-\frac{2 M}{r^{n-1}}-\frac{2 \Lambda r^{2}}{n(n+1)},
$$

$M$ the mass and $\Lambda$ the cosmological constant. For $\Lambda<0$, $M>0$ and any $\kappa, f$ grows monotonically for $r \in(0, \infty)$ from minus to plus infinity, with a simple zero at $r=r_{h}$ and

$$
f=\kappa-\frac{\left(\kappa r_{h}^{(n-1)}-\frac{2 \Lambda r_{h}{ }^{n+1}}{n(n+1)}\right)}{r^{n+1}}-\frac{2 \Lambda r^{2}}{n(n+1)} .
$$

The static region corresponds to $r_{h}<r<\infty$, where we define $x$ as in (5) and find that

$$
x \simeq \begin{cases}\frac{1}{f^{\prime}\left(r_{h}\right)} \ln \left(\frac{r}{r_{h}}-1\right), & r \rightarrow r_{h}^{+} \\ \frac{n(n+1)}{2 \Lambda r}, & r \rightarrow \infty .\end{cases}
$$

In terms of $x$ the static region metric is

$$
d s^{2}=f\left(-d t^{2}+d x^{2}\right)+r^{2} \hat{g}_{A B}(z) d z^{A} d z^{B} .
$$

As for $\mathrm{SAdS}_{4}, x \in(-\infty, 0)$, then the static region manifold $\mathbb{R}_{t} \times(-\infty, 0)_{x} \times \sigma^{n}$ has the causal structure of $\sigma^{n}$ times $a$ half of $1+1$ Minkowski spacetime.

Alternative theories of gravity, such as Lovelock's, admit asymptotically (A)dS black hole solutions with metrics of the form (8) (see [6] and references therein). The function $f$ is no longer given by (9), but in the asymptotically AdS case, by definition, $f \sim-\Lambda_{\text {eff }} r^{2}$ for large $r$ and some negative effective cosmological constant $\Lambda_{\text {eff }}$, whereas near the event horizon $r=r_{h}$ (the largest positive root of $f$ ) $f \sim f^{\prime}\left(r_{h}\right)\left(r-r_{h}\right)$; thus, the integral (5) that defines $x$ [so that (12) holds] diverges logarithmically as $r \rightarrow r_{h}^{+}$and converges for $r \rightarrow \infty$, then $x$ is again restricted to a half line. This is very different to what happens in the asymptotically flat case, where $f \sim 1$ for large $r$, then $x \sim r$ 
in this limit and $x \in(-\infty, \infty)$. Similarly, in the asymptotically de Sitter case, the static region corresponds to $r_{h}<$ $r<r_{c}\left(r_{c}\right.$ is the cosmological horizon). Here $r_{h}$ and $r_{c}$ are simple roots of $f$ that introduce logarithmic divergences near both horizons in the integral defining $x$, therefore $x \in(-\infty, \infty)$. The metric can always be put in the form (12), but only in the asymptotically AdS case is $x$ restricted to a half line and the static region fails to be globally hyperbolic.

For metrics of the form (8) with constant curvature horizons $\sigma^{n}$, scalar and Maxwell fields, as well as linear metric perturbations, can all be expanded as a series in a basis of eigentensors of the Laplace-Beltrami (LB) operator on $\sigma^{n}$, with "coefficients" that carry tensor indexes in the $(t, r)$ Lorentzian orbit manifold [5,7]. We call each term in this series a field mode. After some work, the (Maxwell, linear gravity, etc.) field equations reduce in every case to an infinite set of $1+1$ wave equations for a master variable, one for each mode, with a time-independent potential. Since the massless wave equation in $1+1$ dimension is conformally invariant, the master equation satisfied by the master variable has the Minkowskian form

$$
\left[-\frac{\partial^{2}}{\partial t^{2}}+\frac{\partial^{2}}{\partial x^{2}}-V(x)\right] \phi=0, \quad x<0 .
$$

For GR in arbitrary dimensions, this reduction was proved by Kodama and Ishibashi [5,7]. For black holes with constant curvature horizons in the restricted case of second-order Lovelock theories known as EinsteinGauss-Bonnet gravity, the modal reduction of linear gravity to the form (13) was done in [8,9]. Generalizations to higher order Lovelock theories can be found in [10]. Further examples of reduction of linear field equations to mode equations of the form (13) include hairy black holes, as in [11]. In all these cases, it is the warped product form of the background (8) that allows separation of variables, independently of the form of $f$. The horizon manifold drops out from the field equations, leaving a trace of it in the mode counting (modes are in one-one relation with the eigenspaces of the LB operator on different kinds of tensor fields on $\sigma^{n}$ ), and on the form of the potentials $V$ for each mode. The nonglobally hyperbolic character of the spacetime is what implies the existence of a conformal timelike boundary at $x=0$, contrary to what happens for $\Lambda \geq 0$, for which the domain of the wave equations (13) is $1+1$ Minkowski spacetime, in the asymptotically AdS case $x<0$.

For perturbations propagating in the inner region of a Reissner-Nordström black hole or on the negative mass Schwarzschild or super extreme Reissner-Nordström nakedly singular spaces, the modal decomposition leads again to the $1+1$ wave equation (13) on a half-space. However, in these cases, due to the background curvature singularity, there is a unique self-consistent choice of boundary condition, and thus no ambiguity at all in the dynamics.

Since $V$ in Eq. (13) does not depend on $t$, we can solve it by separating the $t$ variable, after which the problem reduces to finding the eigenvalues and eigenfunctions of a quantum Hamiltonian on a half-line:

$$
\mathcal{H}=-\partial^{2} / \partial x^{2}+V(x), \quad x<0 .
$$

In this paper we study the self-adjoint extensions of the operator defined in (14) and the corresponding solutions of Eq. (13). We do this for the case in which $V$ is continuous for $x \in(-\infty, 0]$ since this case covers the gravitational perturbations of $\mathrm{SAdS}_{4}$ we are interested in. We focus on the different dynamics that arise under homogeneous Dirichlet

$$
\left.\phi\right|_{x=0}=0,
$$

Neumann

$$
\left.\partial_{x} \phi\right|_{x=0}=0
$$

or Robin

$$
\left.\partial_{x} \phi\right|_{x=0}=\left.\gamma \phi\right|_{x=0}
$$

boundary conditions, which are the natural ones, as they preserve the action of spacetime isometries on the solution space (see [12]), with an accent on the least studied Robin boundary condition, for which we find that there is always a range of the Robin parameter $\gamma$ under which the dynamics is unstable. These different choices give all possible selfadjoint extensions of (14).

It is a nontrivial fact that, as far as we know, for scalar and Maxwell fields and also for linearized gravity in four and higher dimensional GR and Lovelock theories with a timelike boundary, $V \rightarrow 0$ as $x \rightarrow-\infty$ and either $V$ is continuous for $x \in(-\infty, 0]$, or $V$ is continuous in $(-\infty, 0)$ and diverges at the conformal boundary always as $V \sim c / x^{2}$ for some constant $c$ that depends on the field type and the mode. For those potentials with $c<3 / 4$ some generalized forms of Robin, Dirichlet and Neumann homogeneous boundary conditions can be defined, whereas if $c \geq 3 / 4$, only homogeneous Dirichlet boundary conditions are allowed [13].

The paper is organized as follows: Sec. II contains some preliminary material on modal decomposition of linear fields and self-adjoint extensions of operators like (14). Our results are gathered in Secs. III and IV. In Sec. III we prove that for nonsingular potentials the $1+1$ wave equation has a critical value of the Robin parameter above which the system is unstable. We show that a boundary condition allowing energy flow from the boundary is not a sufficient condition for instability, and that the instability is due to the 
excitation of a unique negative energy bound state of the associated quantum Hamiltonian, of which we give a number of properties. These results are collected in Propositions 1-6 and illustrated with two toy models. Although motivated by the study of linear gravity on $\mathrm{AdS}_{4}$, these results apply to any problem reducing to equation (13) on a half-space with a nonsingular potential, as well as to the related quantum mechanical problem on a half line.

Section IV explores the effects of the Robin instability of Maxwell fields and gravitational perturbations on $\mathrm{SAdS}_{4}$ with a focus on how the boundary conditions spoil the even-odd duality, which is peculiar of four dimensions and is key in proving the nonmodal stability of the Schwarzschild and Schwarzschild-de Sitter black holes. We show in Proposition 7 that this duality, which is reminiscent of supersymmetric quantum mechanics, is broken except for two out of the infinitely many choices of boundary conditions, and that only one of these gives stable dynamics.

Except for the self-contained section on the scalar field on $\mathrm{SAdS}_{4}$, linear fields in asymptotically AdS backgrounds leading to singular potentials in their modal decompositions are not treated in this work. The massive scalar field on a warped background (8) leads to singular potentials after mode decomposition. For a comprehensive nonmodal study of the massive scalar field on a general [i.e., not necessarily of the form (8)] asymptotically AdS background, the reader is addressed to references [14-18].

\section{DYNAMICS IN A NONGLOBALLY HYPERBOLIC BACKGROUND}

The subject of dynamics in a nonglobally hyperbolic static background has been treated in the series of papers $[12,13,19]$. This is reviewed in Sec. II A, where we explain the relation between boundary conditions for the generalized eigenfunctions of (14) at $x=0$ (i.e., the different selfadjoint extensions of the operator $\mathcal{H}$ ) and the corresponding dynamics driven by (13), which is the equation obeyed by a linear field mode. The modal decomposition of linear fields on the static warped backgrounds (8) in arbitrary dimensions is treated in [5,7] and references therein; [20] offers a detailed description of the four-dimensional case with spherical symmetry. Linear fields are decomposed in independent modes and a master variable is obtained for each mode which satisfies a $1+1$ wave equation of the form (13). In Sec. II B we explain how this is done for linear fields on $\mathrm{SAdS}_{4}$.

\section{A. Boundary conditions and self-adjoint extensions}

After expanding in modes a linear field, the field equations reduce to a set of equations, one for each mode, of the form [see (13) and (14)]

$$
-\ddot{\phi}=\mathcal{H} \phi,
$$

where a dot means time derivative and the Hamiltonian operator is

$$
\mathcal{H}=-\frac{\partial^{2}}{\partial x^{2}}+V(x),
$$

with $V$ continuous in $(-\infty, 0)$ and $V(x) \rightarrow 0$ as $x \rightarrow-\infty$.

The general solution of (15) is of the form

$$
\phi=\int d E c_{E}(t) \psi_{E}(x), \quad \ddot{c}_{E}+E c_{E}=0,
$$

where the $\psi_{E}$ are generalized eigenfunctions

$$
{ }^{z} \mathcal{H} \psi_{E}=E \psi_{E}
$$

of a chosen self-adjoint extension ${ }^{z} \mathcal{H}$ with domain a linear subset of $L^{2}((-\infty, 0), d x)$. In what follows we will use $\mathcal{H}$ to denote the operator (16) acting on unspecified functions, and denote partic ular self-adjoint extensions to specified domains in $L^{2}((-\infty, 0), d x)$ with a left upper superscript. Note that whenever $\mathcal{H}$ admits different self-adjoint extensions, the resulting dynamics from initial compact supported data (which are suitable for all extensions) will depend on the extension we choose.

According to (17), a negative energy $E<0$ in the spectrum of the chosen extension ${ }^{z} \mathcal{H}$ allows exponentially growing terms in (17). Thus, there will be an instability whenever the spectrum of $z \mathcal{H}$ contains a negative $E$ value. The possible self-adjoint extensions depend on the behavior of $V$ near $x=0$ and minus infinity. The potential $V$ is said to be limit circle case (LC) at $x=0$ if any function in the two-dimensional space of local solutions of the differential equation (18) is square-integrable near zero, and is otherwise said to be limit point case (LP) at $x=0$. The same notion applies at minus infinity (see the appendix to section X.I in [21]). It is a nontrivial fact that, according to Theorem X.6 b in [21], the LC/LP notion does not depend on the value of $E$ in (18) as long as $V$ is continuous for $x \in(-\infty, 0)$.

The potentials that appear in the modal decompositions of linear fields are always LP at $x=-\infty$. Some of them are LC at $x=0$, and some others are LP at $x=0$. In the first case, any solution of (18) is of the form $\psi=A \psi_{1}+B \psi_{2}$, where both $\psi_{1}$ and $\psi_{2}$ are square-integrable near $x=0$. Writing $(A, B)=C(\cos (\alpha), \sin (\alpha))$ and allowing the irrelevant overall factor $C$ to be positive or negative while restricting $\alpha \in(-\pi / 2, \pi / 2]$, we find that $\alpha$ parametrizes the set of allowed boundary conditions at $x=0$. If ${ }^{\alpha} \mathcal{H}$ is the operator (16) with domain the linear subset of $L^{2}((-\infty, 0), d x)$ of functions with boundary condition at $x=0$ consistent with choosing $\alpha$ above, then ${ }^{\alpha} \mathcal{H}$ is selfadjoint. Any self -adjoint extension of $\mathcal{H}$ is of this form. 
Thus, if $V$ is LC at $x=0$, there are infinitely many selfadjoint extensions, and the dynamics are unambiguous only after selecting a self-adjoint extension $\alpha$. On the other hand, if $V$ is LP at $x=0$ there is no such ambiguity as there is a single choice of boundary condition under which $\mathcal{H}$ is selfadjoint. In this case we have a unique evolution of initial data at a $t$ slice in spite of the nonglobally hyperbolic character of the outer static region. This is ultimately due to the fact that we have constrained boundary conditions at $x=0$ to those making $\mathcal{H}$ self-adjoint. In principle, there is a much wider set of possible boundary conditions. However, if we require that the time translation symmetry acts in the expected way on the space of solutions and that there is a conserved energy, we are forced to adopt solutions of the form (17) for some self-adjoint extension of $\mathcal{H}$ [12]. Thus, the possibilities for linear fields evolving on the outer static regions of AdS black holes are that either there is a unique dynamic (corresponding to the case where each $V$ is LP at $x=0$ ) or a set of dynamics parametrized by $\alpha \in(-\pi / 2, \pi / 2]$ for each mode such that $V$ is LC at $x=0$.

For massless scalar, Maxwell and gravitational perturbations on $\mathrm{SAdS}_{4}$, the behavior of the mode potentials near the horizon is

$V=f(r)\left[U_{o}+\mathcal{O}\left(r-r_{h}\right)\right], \quad U_{o} \neq 0, \quad r \simeq r_{h}$.

In this limit

$$
x \simeq \frac{\ln \left(\frac{r}{r_{h}}-1\right)}{f^{\prime}\left(r_{h}\right)}
$$

therefore, (19) gives an exponential decay of $V$ as $x \rightarrow-\infty$ [note that $f^{\prime}\left(r_{h}\right)>0$ ],

$$
V \simeq f^{\prime}\left(r_{h}\right) r_{h} e^{f^{\prime}\left(r_{h}\right) x}\left[U_{o}+\cdots\right] .
$$

The local Frobenius series solution of the differential equation (18) near $r=r_{h}$ is, using (19),

$$
\begin{aligned}
\psi_{E}= & C\left(r-r_{h}\right)^{\frac{\sqrt{f^{\prime}}\left(r_{h}\right.}{2}}\left[1+\mathcal{O}\left(r-r_{h}\right)\right] \\
& +D\left(r-r_{h}\right)^{-\frac{\sqrt{f^{\prime}}}{\left(r_{h}\right.}}\left[1+\mathcal{O}\left(r-r_{h}\right)\right] .
\end{aligned}
$$

From (20),

$$
\left(r-r_{h}\right)^{ \pm \frac{\sqrt{-E}}{f^{\prime}\left(r_{h}\right)}} \simeq r_{h}^{\frac{ \pm \sqrt{-E}}{f^{\prime}\left(r_{h}\right)}} e^{ \pm \sqrt{-E x}}
$$

therefore, for positive $E$ any solution (22) is oscillatory, which (as for quantum mechanic wave functions) is a suitable behavior for a generalized eigenfunction, even though it is not square-integrable near minus infinity under $d x$. For negative $E$ the exponentially growing solution has to be discarded, leaving only the $D=0$ solution (22), which decays exponentially with $x$ as $x \rightarrow-\infty$. For no value of $E$ is every function in the two-dimensional space of local solutions of the differential equation (18) squareintegrable near $x=-\infty$ : for negative $E$ the subspace of square-integrable local solutions is one-dimensional and for positive $E$ is trivial [as explained above, since $V$ is continuous for $x \in(-\infty, 0)$, there is no need to check all cases: Theorem X.6 b in [21] guarantees that if the space of local square integrable solutions is less than twodimensional for one value of $E$, it will be so for any $E$ in the complex plane]. Thus, according to the definition above, $V$ is LP at $x=-\infty$. For fields on higherdimensional GR or Lovelock backgrounds we find a similar pattern near the horizon.

In the limit $r \rightarrow \infty$ we find that for Maxwell and gravitational fields on $\mathrm{SAdS}_{4}, V$ is continuous for $x \in(-\infty, 0]$, with

$$
V(x)=v_{0}+v_{1} x+v_{2} x^{2}+\cdots, \quad\left(v_{0} \neq 0\right)
$$

for $x \simeq 0$. The modes of a massless scalar field on $\mathrm{SAdS}_{4}$ instead diverge near $x=0$ as $V \sim 2 / x^{2}$.

For fields on higher-dimensional GR or Lovelock backgrounds we find similarly that either $V$ is nonsingular at $x=0$ or diverges as $V \sim c / x^{2}$.

When analyzing the local solutions of (18) near $x=0$ for both the nonsingular and the $V \sim c / x^{2}$ potentials, we find that $E$ appears only in subleading terms. Thus, any statement of local integrability near $x=0$ is independent of $E$, as anticipated by Theorem X.6 b in [21].

\section{B. Mode decomposition of linear fields on $\mathbf{S A d S}_{4}$}

In this section we illustrate the modal decomposition for the Klein-Gordon and Maxwell fields and for linear metric perturbations on the $\mathrm{SAdS}_{4}$ background (see, e.g., [20]). For a systematic treatment of the modal decomposition on dimension $D \geq 4$ black holes with constant curvature horizon manifolds we refer the reader to [5,7]. As explained in the paragraph above Eq. (13), the word mode in this context applies to the individual terms when writing scalar and tensor fields as a series in a basis of eigenscalar/ tensors of the LB operator of the constant curvature horizon manifold. In the case of $\mathrm{SAdS}_{4}$, this manifold is the unit sphere and a mode corresponds to a fixed $(\ell, m)$ harmonic solution of the field equations. Modes can be further decomposed into their time Fourier components $(\ell, m, \omega)$ of frequency $\omega$.

In all cases the modal decomposition reduces the problem of linear fields propagating on a background (12) to a set of $1+1$ wave equations of the form (13), independently of the dimension of the background.

\section{Massless scalar fields on $\mathbf{S A d S}_{4}$}

For the $\mathrm{SAdS}_{4}$ metric (1)-(2), the massless scalar field equation reads 


$$
\begin{aligned}
0= & \nabla_{\alpha} \nabla^{\alpha} \Phi=(r f)^{-1}\left[-\partial_{t}^{2}+f \partial_{r}\left(f \partial_{r}\right)\right. \\
& \left.+f\left(r^{-2} \Delta-f^{\prime} / r\right)\right](r \Phi),
\end{aligned}
$$

where $\Delta$ is the Laplacian on the unit sphere

$$
\Delta=\partial_{\theta}^{2}+\cot (\theta) \partial_{\theta}+\sin (\theta)^{-2} \partial_{\varphi}^{2} .
$$

Introducing

$$
\phi=r \Phi
$$

and expanding $\phi$ in an $L^{2}\left(S^{2}\right)$ orthonormal basis $S_{(\ell, m)}$ of real spherical harmonics on the sphere,

$$
\phi=\sum_{(\ell, m)} \phi_{(\ell, m)}(t, x) S_{(\ell, m)}(\theta, \phi),
$$

we find that (25) is equivalent to a set of wave equations of the form (15) in the $x<0$ half of $1+1$ Minkowski spacetime,

$$
-\ddot{\phi}_{(\ell, m)}=\mathcal{H}_{\ell}^{s} \phi_{(\ell, m)},
$$

with Hamiltonian

$$
\mathcal{H}_{\ell}^{s}=-\frac{\partial^{2}}{\partial x^{2}}+V_{\ell}^{s}
$$

and potential

$$
V_{\ell}^{s}=f\left[\frac{2 M}{r^{3}}+\frac{\ell(\ell+1)}{r^{2}}-\frac{2}{3} \Lambda\right] .
$$

Note that $V_{\ell}^{s}>0$ and that

$$
V_{\ell}^{s} \sim \begin{cases}\frac{2}{x^{2}}-\frac{\Lambda}{3}\left(\ell^{2}+\ell+2\right)+\mathcal{O}(x), & \text { as } x \rightarrow 0^{-} \\ \left(\ell(\ell+1)+1-\Lambda r_{h}^{2}\right)\left(1-\Lambda r_{h}^{2}\right) r_{h}^{-2} \exp \left(\left(\frac{1-\Lambda r_{h}^{2}}{r_{h}}\right) x\right), & \text { as } x \rightarrow-\infty\end{cases}
$$

According to Theorem X.10 in [21], the fact that the $x^{-2}$ coefficient in (32) is greater than $3 / 4$ implies that the potential $V_{e}^{s}$ belongs to the limit point case at $x=0$ (see Sec. II A), which means that the space of local solutions of

$$
\left(-\partial_{x}^{2}+V_{\ell}^{s}\right) \psi=E \psi
$$

for which $\int_{c}^{0} \psi^{2} d x<\infty$ for some negative $c$ is onedimensional. To check this, note that the two-dimensional space of Frobenius series solutions for (33) at $x=0$ $(r=\infty)$ is

$$
\begin{aligned}
\psi= & A\left(\frac{1}{r^{2}}-\frac{3}{10 \Lambda^{2}} \frac{(\ell+3)(\ell-2) \Lambda+3 E}{r^{4}}+\mathcal{O}\left(r^{-5}\right)\right) \\
& +B\left(r+\frac{3}{2 \Lambda^{2}} \frac{\ell(\ell+1) \Lambda+3 E}{r}+\mathcal{O}\left(r^{-2}\right)\right),
\end{aligned}
$$

and $B=0$ is required for the integral $\int_{r_{o}}^{\infty} \psi^{2} d r / f$ to converge. Since there is a unique admissible condition at $x=0$, namely, $B=0$ in (34) (i.e., Dirichlet), we conclude that the dynamics of massless scalar fields on $\mathrm{SAdS}_{4}$ are not ambiguous. The extension of the domain of $\mathcal{H}_{\ell}^{s}=$ $-\partial_{x}^{2}+V_{\ell}^{s}$ from functions of compact support to allow $\psi \sim r^{-2}$ for large $r[B=0$ in (34)] gives a self-adjoint operator which, as we will now show, has a positive spectrum.

For $x \rightarrow-\infty\left(r \rightarrow r_{h}^{+}\right)$,

$$
\begin{aligned}
\psi= & C\left(r-r_{h}\right)^{\kappa}\left(1+\mathcal{O}\left(r-r_{h}\right)\right) \\
& +D\left(r-r_{h}\right)^{-\kappa}\left(1+\mathcal{O}\left(r-r_{h}\right)\right), \quad \kappa=\frac{\sqrt{-E r_{h}^{2}}}{1-\Lambda r_{h}^{2}},
\end{aligned}
$$

[compare with (22)] where it is assumed that we take the real part.

If $E>0$, a solution behaving like (34) with $B=0$ at infinity will behave near the horizon as in (35). This oscillatory behavior is characteristic of generalized eigenfunctions for potentials that vanish in this limit, Eq. (32).

However, a negative value of $E$ [real positive $\kappa$ in (35)] would be admissible only if there were solutions of (33) behaving as in (34) with $B=0$ near $x=0$ and as in (35) with $D=0$ near the horizon. To show that this is not possible, note that, assuming $B=D=0$ and $E<0$, $\int_{-\infty}^{0} \psi^{2} d x$ converges and we arrive at a contradiction,

$$
\begin{aligned}
E \int_{-\infty}^{0} \psi^{2} d x & =\int_{-\infty}^{0} \psi\left(-\partial_{x}^{2} \psi+V_{\ell}^{s} \psi\right) d x=\left.\left[\psi f \partial_{r} \psi\right]\right|_{r=r_{h}} ^{r=\infty}+\int_{-\infty}^{0}\left(\left(\partial_{x} \psi\right)^{2}+V_{\ell}^{s} \psi^{2}\right) d x \\
& =\int_{-\infty}^{0}\left(\left(\partial_{x} \psi\right)^{2}+V_{\ell}^{s} \psi^{2}\right) d x>0 .
\end{aligned}
$$


We conclude that the dynamics of massless scalar fields on $\mathrm{SAdS}_{4}$ are free of ambiguities, as there is a unique possible self-adjoint extension ${ }^{D} \mathcal{H}_{\ell}^{s}$ (for Dirichlet) of $\mathcal{H}_{\ell}^{s}$ [that allowing only $B=0$ in (34)], and that the field has no modal instabilities, as for every $\ell$ the spectrum of ${ }^{D} \mathcal{H}_{\ell}^{s}$ has no negative eigenvalues.

Stronger results on the stability of scalar fields on asymptotically AdS backgrounds can be found in [14-18], where boundedness and decay were studied using a nonmodal approach to the Klein-Gordon equation.

Maxwell fields, as well as gravitational perturbations, exhibit more complicated patterns: the effective potentials of the modes are nonsingular at $x=0$ and this allows for infinitely many boundary conditions, some of them leading to unstable dynamics. To show this, we need to recall how to reduce Maxwell equations and the linearized Einstein equations (LEE) to a set of the form (15)-(16) using tensor decompositions into harmonic $S^{2}$ tensors, generalizing what was done in (28) for the scalar field. This is a well-known procedure which, for $D=4$, is reviewed, e.g., in $[2,20]$ and specifically for $\mathrm{SAdS}_{4}$ in [22].

\section{Maxwell fields on $\mathbf{S A d S}_{\mathbf{4}}$}

Write the Maxwell potential as a sum of its vector/odd (-) and scalar/even (+) pieces, $A_{\beta}=A_{\beta}^{(-)}+A_{\beta}^{(+)} \quad[2,20,22]$; these can be gauge fixed to the form
$A_{\beta}^{(-)}=\sum_{(\ell, m)} \phi^{(-, \ell, m)}\left(0,0, \frac{1}{\sin \theta} \partial_{\phi} S_{(\ell, m)},-\sin \theta \partial_{\theta} S_{(\ell, m)}\right)$

$A_{\beta}^{(+)}=\sum_{(\ell, m)}\left(f \partial_{r} \phi^{(+, \ell, m)} S_{(\ell, m)}, f^{-1} \partial_{t} \phi^{(+, \ell, m)} S_{(\ell, m)}, 0,0\right)$,

where $\phi^{( \pm, \ell, m)}$ are functions of $(t, r)$. Maxwell equations $F=d A$ and $\nabla^{\alpha} F_{\alpha \beta}=0$ are equivalent to

$$
-\ddot{\phi}_{(\ell, m)}^{ \pm}=\mathcal{H}_{\ell}^{\mathrm{Max}} \phi_{(\ell, m)}^{ \pm},
$$

where the Hamiltonian

$$
\mathcal{H}_{\ell}^{\operatorname{Max}}=-\partial_{x}^{2}+V_{\ell}^{\operatorname{Max}}
$$

is independent of the \pm parity and the azimuthal number $m$ and has a potential

$$
V_{\ell}^{\operatorname{Max}}=f \frac{\ell(\ell+1)}{r^{2}} .
$$

Note that $V_{\ell}^{\operatorname{Max}}>0$ and that

$$
V_{\ell}^{\mathrm{Max}} \sim \begin{cases}-\frac{\Lambda}{3} \ell(\ell+1)+\mathcal{O}\left(x^{2}\right), & \text { as } x \rightarrow 0^{-} \\ \ell(\ell+1)\left(1-\Lambda r_{h}^{2}\right) r_{h}^{-2} \exp \left(\left(\frac{1-\Lambda r_{h}^{2}}{r_{h}}\right) x\right), & \text { as } x \rightarrow-\infty .\end{cases}
$$

For future reference we also note that

$$
\int_{-\infty}^{0} V_{\ell}^{\operatorname{Max}} d x=\int_{r_{h}}^{\infty} V_{\ell}^{\operatorname{Max}} \frac{d r}{f}=\frac{\ell(\ell+1)}{r_{h}} .
$$

\section{Gravitational waves on $\mathrm{SAdS}_{\mathbf{4}}$}

As done for Maxwell fields, we can decompose metric perturbations into scalar/even/+ and vector/odd/ - fields with harmonic numbers $(\ell, m)$. This procedure is well known; the details can be found in [2,20] and references therein. The $\ell=0,1$ sectors contain either pure gauge fields or time-independent fields, which corresponds to perturbations within the Kerr family, that is, a variation of mass or an addition of angular momentum. These are irrelevant to the stability problem, so we will focus on the $\ell \geq 2$ modes.

For,$\pm \ell \geq 2$ modes the LEE reduce to the well-known Regge-Wheeler (-) and Zerilli (+) equations for the gauge invariant fields $\phi_{(\ell, m)}^{ \pm}(t, r)$, which are of the form (15)-(16). The Regge-Wheeler potential of the odd Hamiltonian $\mathcal{H}_{\ell}^{-}$is

$$
V_{\ell}^{(-)}=f\left(\frac{\ell(\ell+1)}{r^{2}}-\frac{6 M}{r^{3}}\right),
$$

and is positive in the outer static region $r>r_{h}$ except when $r_{h}$ is small compared to $M$, in which case it is negative in the interval $r_{h}<r<6 M /(\ell(\ell+1))$. This potential behaves as

$$
V_{\ell}^{(-)} \sim \begin{cases}-\Lambda \ell(\ell+1) / 3+\mathcal{O}(x), & \text { as } x \rightarrow 0^{-} \\ \left(\ell(\ell+1) r_{h}-6 M\right)\left(1-\Lambda r_{h}^{2}\right) r_{h}^{-3} \exp \left(\left(\frac{1-\Lambda r_{h}^{2}}{r_{h}}\right) x\right), & \text { as } x \rightarrow-\infty .\end{cases}
$$


The Zerilli potential of the even Hamiltonian $\mathcal{H}_{\ell}^{+}$is

$$
V_{\ell}^{(+)}=f \frac{\left[\mu^{2} \ell(\ell+1)-24 M^{2} \Lambda\right] r^{3}+6 \mu^{2} M r^{2}+36 \mu M^{2} r+72 M^{3}}{r^{3}\left(6 M+\mu^{2} r\right)^{2}}, \quad \mu=(\ell-1)(\ell+2),
$$

this potential is positive for $r>r_{h}$ and behaves as

$$
V_{\ell}^{(+)} \sim \begin{cases}\left(24 M^{2} \Lambda^{2}\right) \mu^{-2}-\Lambda \ell(\ell+1)+\mathcal{O}(x), & \text { as } x \rightarrow 0^{-} \\ \frac{\left(\Lambda^{2} r_{h}^{4}-4 \Lambda r_{h}^{2}+\ell^{4}+2 \ell^{3}-\ell^{2}-2 \ell+3\right)\left(1-\Lambda r_{h}^{2}\right)}{\left(1-\Lambda r_{h}^{2}+\ell(\ell+1)\right) r_{h}^{2}} \exp \left(\left(\frac{1-\Lambda r_{h}^{2}}{r_{h}}\right) x\right), & \text { as } x \rightarrow-\infty .\end{cases}
$$

For future reference we note that

$$
\int_{-\infty}^{0} V_{\ell}^{(+)} d x=\frac{2 \Lambda^{2} r_{h}{ }^{3}}{3(\ell+2)(\ell-1)}+\frac{\Lambda r_{h}(\ell+3)(\ell-2)}{2(\ell+2)(\ell-1)}+\frac{2 \ell^{2}+2 \ell-3}{2 r_{h}} .
$$

\section{NONSINGULAR POTENTIALS AND ROBIN INSTABILITIES}

By a nonsingular potential $V$ in (16) we mean one that is continuous for $x \in(-\infty, 0]$, satisfies (19) and, for $x \simeq 0$,

$$
V(x)=v_{0}+v_{1} x+v_{2} x^{2}+\cdots, \quad\left(v_{0} \neq 0\right) .
$$

In this case, the local solutions near $x=0$ of the differential equation (18) are of the form

$$
\psi_{E}=K \cos (\alpha)\left[1+\left(\frac{v_{o}-E}{2}\right) x^{2}+\frac{v_{1}}{6} x^{3}+\mathcal{O}\left(x^{4}\right)\right]+K \sin (\alpha)\left[x-\left(\frac{v_{o}-E}{6}\right) x^{3}+\mathcal{O}\left(x^{4}\right)\right],
$$

where $A=K \cos (\alpha)=\psi_{E}(0)$ and $B=K \sin (\alpha)=\psi_{E}^{\prime}(0)$, and we choose to allow negative values of $K$ and restrict $\alpha \in(-\pi / 2, \pi / 2$ ]. As discussed above [see the paragraph starting at Eq. (19)], the potential is LP at $x=-\infty$. From (50) it follows that the potential is LC at $x=0$, with boundary conditions parametrized by $\alpha$. Note that $\psi_{E}(0)$ and $\partial_{x} \psi_{E}(0)$ are well defined and that the possible boundary conditions at $x=0$ are Dirichlet $(\alpha=\pi / 2)$, Neumann $(\alpha=0)$ or Robin (the remaining cases). Robin boundary conditions are characterized by the nonzero value of

$$
\psi_{E}^{\prime}(0) / \psi_{E}(0)=\tan (\alpha) \equiv \gamma .
$$

Restricting to eigenfunctions satisfying (50) with a fixed $\alpha$ value defines the spectrum of a self-adjoint extension of $\mathcal{H}$. We denote the corresponding self-adjoint Hamiltonian operator for $\alpha=\pi / 2, \alpha=0$ and $\gamma=\tan (\alpha) \neq 0, \infty$ respectively as ${ }^{D} \mathcal{H},{ }^{N} \mathcal{H}$ and ${ }^{\gamma} \mathcal{H}$.

In this section we study the possibility that a chosen selfadjoint extension $z \mathcal{H}$ admits a negative energy eigenfunction $\psi_{E}, E<0$, and establish a number of properties for such a state. The results of this section apply then to the quantum mechanics problem on a half line with a nonsingular potential (as defined above) as well as any $1+1$ wave equation (15)-(16) with such a potential, for which, as explained above, a negative energy eigenvalue implies, in virtue of Eq. (17), that a generic solution of the $1+1$ wave equation grows exponentially in time. Our motivation is, of course, spotting such instabilities in Maxwell fields and linearized gravity in $\mathrm{SAdS}_{4}$, whose modal decompositions lead to the nonsingular potentials (41), (44) and (46).

The asymptotic behavior near the horizon of a negative energy eigenfunction $\psi_{E}$,

$-\psi_{E}^{\prime \prime}+V \psi_{E}=E \psi_{E}, \quad$ for $x \in(-\infty, 0), \quad E<0$,

is that in (22) with $D=0$, then $\psi_{E}$ is a "bound state," that is, belongs to $L^{2}((-\infty, 0), d x)$ [as follows from (22) and (23)],

$$
\int_{-\infty}^{0} \psi_{E}^{2} d x<\infty
$$

If the chosen self-adjoint extension is not Dirichlet, we may normalize $\psi_{E}$ such that

$$
\psi_{E}(0)=1, \quad \psi_{E}^{\prime}(0)=\gamma .
$$

A key observation is that our intuition from quantum mechanics in $\mathbb{R}$ fails for the Schrödinger operator $\mathcal{H}$ on a 
half line subject to Robin boundary conditions, as this may admit negative eigenvalues even if $V \geq 0$. The reason is that the "kinetic energy" operator $-\partial_{x}^{2}$ fails to be positive definite if $\gamma>0$, as the following simple calculation of the expectation values of $\mathcal{H}$ for a (not necessarily normalized) real, square-integrable function on $(-\infty, 0)$ shows:

$$
\begin{aligned}
\langle\psi, \mathcal{H} \psi\rangle:= & \int_{-\infty}^{0} \psi \mathcal{H} \psi d x=-\left.\psi \psi^{\prime}\right|_{x=0} \\
& +\int_{-\infty}^{0}\left[\left(\psi^{\prime}\right)^{2}+V \psi^{2}\right] d x .
\end{aligned}
$$

For Dirichlet or Neumann boundary conditions at $x=0$ the first term on the right vanishes and we find that $V \geq 0$ does imply $\langle\psi, \mathcal{H} \psi\rangle>0$, i.e., the self-adjoint extensions ${ }^{D} \mathcal{H}$ and ${ }^{N} \mathcal{H}$ have positive spectra. For a self-adjoint extension ${ }^{\gamma} \mathcal{H}$ corresponding to the Robin boundary condition

$$
\psi^{\prime}(0)=\gamma \psi(0), \quad \gamma \neq 0,
$$

we find from (55) that

$$
\langle\psi, \gamma \mathcal{H} \psi\rangle=-\gamma \psi(0)^{2}+\int_{-\infty}^{0}\left[\left(\psi^{\prime}\right)^{2}+V \psi^{2}\right] d x,
$$

which, assuming $V \geq 0$, is positive for $\gamma<0$, but may be negative if $\gamma>0$.

\section{A. General results for nonsingular potentials}

In what follows, we use the fact that any function in the domain of a self-adjoint operator such as ${ }^{\gamma} \mathcal{H}$ can be expanded in a basis of generalized eigenfunctions of the operator. Therefore, as in ordinary quantum mechanics, there is a function $\psi$ in this domain for which $\langle\psi, \gamma \mathcal{H} \psi\rangle<0$ if and only if the spectrum of ${ }^{\gamma} \mathcal{H}$ contains a negative value of $E$.

Proposition 1. (Robin instabilities) Let $V$ be nonsingular. For large enough positive $\gamma$ the spectrum of $\gamma \mathcal{H}$ contains a negative eigenvalue.

Proof.-Since nonsingular potentials are bounded, $|V(x)| \leq V_{o}$ for some $V_{o} \geq 0$. Consider now the trial function $\psi=e^{\gamma x}$. If $\gamma>0$, this function belongs to the domain of $\gamma \mathcal{H}$, and the expectation value of $\gamma \mathcal{H}$ is

$$
\begin{aligned}
\langle\psi, \gamma \mathcal{H} \psi\rangle & =\int_{-\infty}^{0} e^{\gamma x}\left(-\gamma^{2} e^{\gamma x}+V e^{\gamma x}\right) d x \\
& =-\frac{\gamma}{2}+\int_{-\infty}^{0} V e^{2 \gamma x} d x \leq-\frac{\gamma}{2}+\frac{V_{o}}{2 \gamma},
\end{aligned}
$$

which is negative for

$$
\gamma>\sqrt{V_{o}} .
$$

Proposition 2. Let $V(x)$ be nonsingular. Assume that $\gamma_{\circ} \mathcal{H}$ admits a negative energy bound eigenstate, then so does $\gamma \mathcal{H}$ for $\gamma>\gamma_{o}$.

Proof.-Let $\psi_{o}$ be a negative energy bound eigenstate of $\gamma_{o} \mathcal{H}$, normalized such that $\psi_{o}(0)=1$, i.e., $\psi_{o}$ satisfies (52)-(54) for $\gamma_{o}$. Fix $\alpha \equiv \gamma-\gamma_{o}>0$ and let $\phi_{\delta}(x)$ be a smooth function of $(\delta, x) \in\left(-\delta_{0}, \delta_{o}\right) \times\left(-\infty, \delta_{o}\right)$ for some positive $\delta_{o}$ such that: $\phi_{\delta}(-\delta)=1, \phi_{\delta}{ }^{\prime}(-\delta)=0=$ $\phi_{\delta}^{\prime \prime}(-\delta), \phi_{\delta}$ and $\phi_{\delta}^{\prime}$ are growing functions for $x \in(-\delta, 0)$, $\phi_{\delta}^{\prime}(0) / \phi_{\delta}(0)=\alpha$ and $\phi_{\delta}(0)<2$. An example of such a function is

$$
\phi_{\delta}(x)=\frac{\alpha(x+\delta)^{3}}{\delta^{2}(3-\alpha \delta)}+1, \quad 0<\delta<\frac{3}{2 \alpha}=\delta_{o} .
$$

Define

$$
\psi_{\delta}(x)= \begin{cases}\psi_{o}(x), & x \leq-\delta \\ \psi_{o}(x) \phi_{\delta}(x), & -\delta<x \leq 0 .\end{cases}
$$

Note that this function belongs to the domain of $\gamma_{\mathcal{H}}$. An example of $\psi_{o}$ and the corresponding $\psi_{\delta}$ is depicted in Fig. 1. Since $V$ is continuous and bounded, $\psi_{o}$ and $\psi_{o}^{\prime}$ are bounded near $x=0$, and we may assume that

$$
\begin{aligned}
\left|\psi_{o}(x)\right| & <A, \quad\left|\psi_{o}^{\prime}(x)\right|<B, \\
|V(x)| & <C \quad \text { for } x \in\left(-\delta_{o}, 0\right) .
\end{aligned}
$$

This implies that

$\left|\psi_{\delta}(x)\right|<2 A, \quad\left|\psi_{\delta}^{\prime}(x)\right|<2(B+\alpha A) \quad$ for $x \in\left(-\delta_{o}, 0\right)$.

From these two equations and $\phi_{\delta}(0)=(1-\alpha \delta / 3)^{-1}$ it follows that

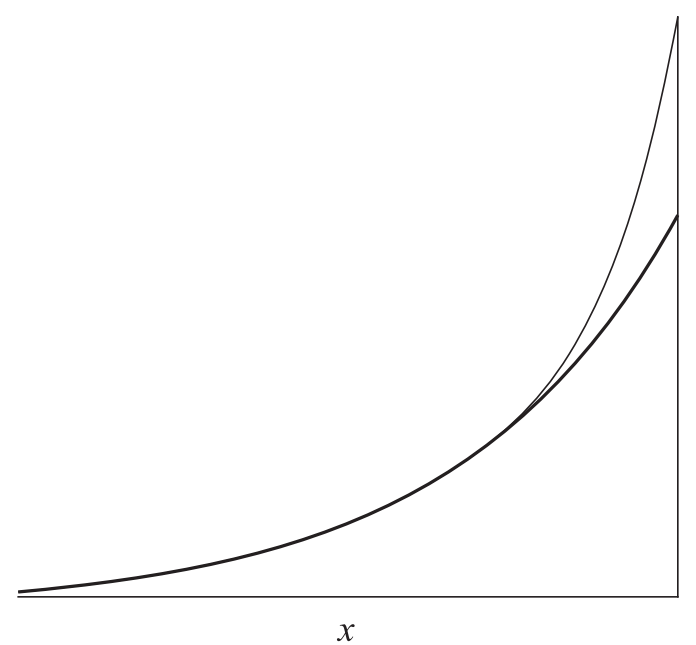

FIG. 1. An example of $\psi_{o}$ (thick line) and the corresponding $\psi_{\delta}$ (thin line) used in Proposition 2. 


$$
\begin{aligned}
& \left\langle\psi_{\delta},{ }^{\gamma \mathcal{H}} \psi_{\delta}\right\rangle-\left\langle\psi_{o},{ }{ }_{o} \mathcal{H} \psi_{o}\right\rangle \\
& =\gamma_{o}-\gamma \phi_{\delta}(0)^{2}+\int_{-\delta}^{0}\left[\left(\psi_{\delta}^{\prime}\right)^{2}-\left(\psi_{o}^{\prime}\right)^{2}+V\left(\psi_{\delta}^{2}-\psi_{o}^{2}\right)\right] \\
& \quad<\gamma_{o}-\gamma(1-\alpha \delta / 3)^{-2}+\delta\left[4(B+\alpha A)^{2}+B^{2}+5 C A^{2}\right] .
\end{aligned}
$$

Note that $A, B$ and $C$ are independent of $\delta$, then for $\delta$ small enough the right side of (64) is negative. This implies that $\left\langle\psi_{\delta},{ }^{\gamma} \mathcal{H} \psi_{\delta}\right\rangle<\left\langle\psi_{o},{ }^{\gamma_{o}} \mathcal{H} \psi_{o}\right\rangle<0$. Since $\psi_{\delta}$ is in the domain of $\gamma \mathcal{H}$, the spectrum of $\gamma_{\mathcal{H}}$ contains a negative energy.

\section{B. Non-negative nonsingular potentials}

For non-negative nonsingular potentials a number of useful properties can be easily proved:

Proposition 3. Let $V(x) \geq 0$ be a nonsingular potential. The self-adjoint extensions ${ }^{D} \mathcal{H},{ }^{N} \mathcal{H}$ and ${ }^{\gamma} \mathcal{H}$ with $\gamma<0$ are positive definite.

Proof.-This follows from Eqs. (55) and (57).

The following proposition shows that if $V(x) \geq 0$ is nonsingular and $\gamma$ positive, $\gamma \mathcal{H}$ admits at most one negative energy eigenstate. It also establishes some properties of the corresponding eigenfunction.

Proposition 4. Let $V(x) \geq 0$ be a nonsingular potential. Assume there is $E<0$ and $\psi_{E}$ satisfying (52)-(54), then it follows that:

(i) $\psi_{E}$ has no roots, then we can choose it to be positive.

(ii) $\psi_{E}$ grows monotonically from 0 to $1=\psi_{E}(0)$ and $\psi_{E}^{\prime}$ grows monotonically from 0 to $\gamma=\psi_{E}^{\prime}(0)$ in the interval $x \in(-\infty, 0]$.

(iii) There is at most one $E<0$ and one $\psi_{E}$ for which conditions (52)-(54) hold.

Proof.

(i) First note that the roots of $\psi_{E}$ are isolated points: if $x_{o}=\lim _{n \rightarrow \infty} x_{n}$ were roots of $\psi_{E}$, then $\psi_{E}\left(x_{o}\right)=$ $0=\partial_{x} \psi_{E}\left(x_{o}\right)$ and, since $\psi_{E}$ satisfies the secondorder equation (52), $\psi_{E}(x)=0$ for all $x$, which is a contradiction. Note also that there cannot exist a sequence $x_{n}$ of consecutive roots such that $\lim _{n \rightarrow \infty} x_{n}=-\infty$; otherwise, there would be a sequence $x_{n}^{\prime}$ of positive local maxima of $\psi_{E}$, $\lim _{n \rightarrow \infty} x_{n}^{\prime}=-\infty$, and/or a sequence $x_{n}^{\prime \prime}$ of negative local minima of $\psi_{E}, \lim _{n \rightarrow \infty} x_{n}^{\prime \prime}=-\infty$. Both cases lead to a contradiction: take, e.g., a sequence $x_{n}^{\prime}$ of positive maxima: the conditions $\psi_{E}\left(x_{n}^{\prime}\right)>0$, $\partial_{x}^{2} \psi_{E}\left(x_{n}^{\prime}\right)<0, \lim _{n \rightarrow \infty} x_{n}^{\prime}=-\infty$ give

$$
0 \geq \lim _{n \rightarrow \infty} \frac{\partial_{x}^{2} \psi_{E}\left(x_{n}^{\prime}\right)}{\psi_{E}\left(x_{n}^{\prime}\right)}=\lim _{j \rightarrow \infty}\left(V\left(x_{n}^{\prime}\right)-E\right)=-E,
$$

which contradicts $E<0$ [if we assume a sequence $x_{n}^{\prime \prime}$ of negative minima we get the same contradiction as, again, $\left.\partial_{x}^{2} \psi_{E}\left(x_{n}^{\prime \prime}\right) / \psi_{E}\left(x_{n}^{\prime \prime}\right)<0\right]$. We may therefore assume, without loss of generality (i.e., replacing $\psi_{E}$ with $-\psi_{E}$ if necessary), that $\psi_{E}>0$ for large negative $x$. Now assume $\psi_{E}$ has roots, then there is a root $x_{o}$ with largest absolute value (the least upper bound of the nonempty bounded set $\{z \leq 0 \mid \psi(x)>0$ for $x \in(-\infty, z)\})$. Since $\psi_{E}$ is square-integrable, $\quad \lim _{x \rightarrow-\infty} \psi_{E}(x)=0=\psi_{E}\left(x_{o}\right)$, and $\psi_{E}(x)>0$ in the interval $x \in\left(-\infty, x_{o}\right)$. It follows that there is a local maximum at $x_{1}<x_{o}$, and this leads us back to the same type of contradiction as above: $0>\partial_{x}^{2} \psi_{E}\left(x_{1}\right) / \psi_{E}\left(x_{1}\right)=V\left(x_{1}\right)-E$; however, $V\left(x_{1}\right)-E>0$.

(ii) As $\psi_{E}$ is in the domain of $\gamma \mathcal{H}, \int_{-\infty}^{0}\left(\psi_{E}^{\prime}\right)^{2} d x<\infty$, then $\lim _{x \rightarrow-\infty} \psi_{E}^{\prime}(x)=0$. From the hypotheses and i) it follows that $\psi_{E}>0$ for $x \in(-\infty, 0]$, then $\partial_{x}^{2} \psi_{E}=(V-E) \psi_{E}>0$. This implies that $\partial_{x} \psi_{E}$ grows monotonically from zero to $\partial_{x} \psi_{E}(0)$. As $\partial_{x} \psi_{E}>0$ everywhere, $\psi_{E}$ increases monotonically from zero to $\psi_{E}(0)$.

(iii) Assume $0>E_{2}>E_{1}$ are two eigenvalues of $\gamma \mathcal{H}$. In view of ii) we may assume that $\psi_{E_{i}}(x)>0$ for all $x$, $i=1,2$. Equation (52) implies that the Wronskian

$$
W=\left(\partial_{x} \psi_{E_{1}}\right) \psi_{E_{2}}-\left(\partial_{x} \psi_{E_{2}}\right) \psi_{E_{1}}
$$

satisfies $\partial_{x} W=\left(E_{2}-E_{1}\right) \psi_{E_{2}} \psi_{E_{1}}>0$ for all $x$. This implies that $W$ grows monotonically. However, $\lim _{x \rightarrow-\infty} W(x)=0=W(0)$. The uniqueness of $\psi_{E}$ follows from (54).

Proposition 5. Let $V(x) \geq 0$ be a nonsingular potential and consider the operator $\gamma \mathcal{H}$ for $\gamma>0$.

(i) If $V$ is nontrivial, for small enough $\gamma$ the spectrum of $\gamma \mathcal{H}$ contains no negative eigenvalue.

(ii) There is a critical value $\gamma_{c}>0$ such that the set $\{\gamma \mid$ the spectrum of $\gamma \mathcal{H}$ contains a negative eigenvalue $\}$ is of the form $\left(\gamma_{c}, \infty\right)$.

(iii) For $\gamma>\gamma_{c}$, the negative energy eigenvalue $E_{\gamma}$ satisfies $\left|E_{\gamma}\right| \leq 2 \gamma^{2}$.

(iv) If $\int_{-\infty}^{0} V d x<\infty$ then $\gamma_{c} \leq 2 \int_{-\infty}^{0} V d x$.

Proof.

(i) Assume ${ }^{\gamma} \mathcal{H}$ admits a negative energy $E_{\gamma}$ and let $\psi_{E_{\gamma}}$ be the eigenstate satisfying $\psi_{E_{\gamma}}(0)=1$. From Lemma 4 we know that $\psi_{E_{\gamma}}$ is positive, convex and monotonically growing in $(-\infty, 0]$. In particular, $\psi_{E_{\gamma}}(x)>\gamma x+1$ for $-1 / \gamma<x<0$ (Fig. 2), then

$$
\begin{aligned}
\gamma= & \int_{-\infty}^{0} \psi_{E_{\gamma}}^{\prime \prime}(x) d x=\int_{-\infty}^{0}\left(V(x)-E_{\gamma}\right) \psi_{E_{\gamma}}(x) d x \\
& >\int_{-1 / \gamma}^{0}\left(V(x)-E_{\gamma}\right)(\gamma x+1) d x=h(\gamma)+k(\gamma),
\end{aligned}
$$

where, for $\gamma>0$ we defined 


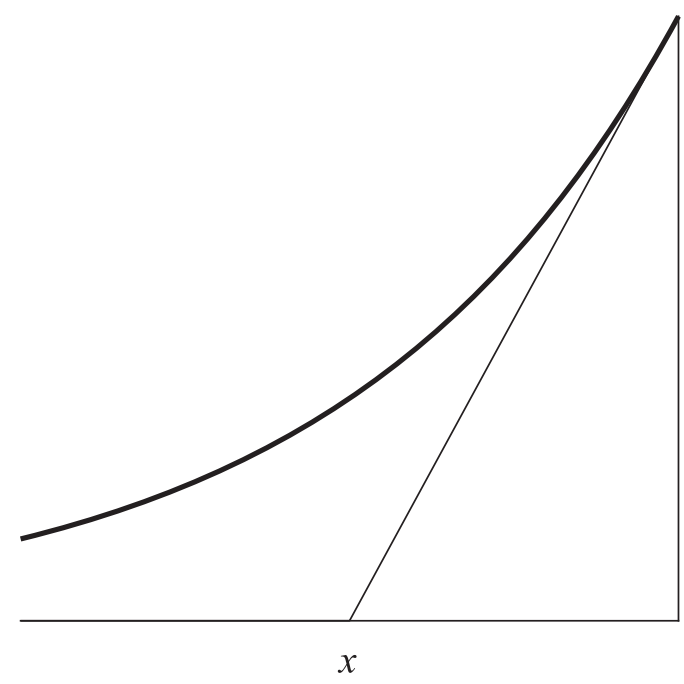

FIG. 2. Graphs of $\psi_{E_{\gamma}}$ (thick line) and $\gamma x+1$ for $x>-1 / \gamma$, zero otherwise (thin line) used in Proposition 5.i.

$$
\begin{aligned}
& h(\gamma)=\int_{-1 / \gamma}^{0} V(x)(\gamma x+1) d x \geq 0 \quad \text { and } \\
& k(\gamma)=-\frac{E_{\gamma}}{2 \gamma}>0 .
\end{aligned}
$$

Note that $d h / d \gamma=\int_{-1 / \gamma}^{0} V(x) x d x \leq 0$, then $h$ is a positive, decreasing function of $\gamma$, diverging as $\gamma \rightarrow 0^{+}$unless $\int_{-\infty}^{0} V(x) d x$ is finite. It follows from (67) and (68) that the existence of a negative energy eigenvalue implies $\gamma>h(\gamma)$, and this implies that $\gamma>\gamma_{*}$, where $\gamma_{*}>0$ is the only solution of $\gamma=h(\gamma)$. We conclude that, for small positive $\gamma,{ }^{\gamma} \mathcal{H}$ is positive definite.

(ii) This follows from i) and Propositions 1 and 2. For $\gamma=\gamma_{c}$ we expect the lowest-energy eigenvalue to be $E=0$.

(iii) From (67) and (68) it follows that, for the bound state, $\gamma \geq k(\gamma)=-E_{\gamma} /(2 \gamma)$.

(iv) For the trial function used in Proposition 1

$$
\langle\psi, \gamma \mathcal{H} \psi\rangle=-\frac{\gamma}{2}+\int_{-\infty}^{0} V e^{2 \gamma x} d x<-\frac{\gamma}{2}+\int_{-\infty}^{0} V d x,
$$

and this is negative for

$$
\gamma>2 \int_{-\infty}^{0} V d x
$$

For potentials with finite integral, condition (70) assures the existence of negative energy states for ${ }^{\gamma} \mathcal{H}$. The sharper bound

$$
\gamma>\int_{-\infty}^{0} V d x
$$

was proved in [11] using different methods. We can prove (71) by making the assumption that the spectrum of ${ }^{\gamma} \mathcal{H}$ is continuous in $\gamma$.

Proposition 6. Let $V(x) \geq 0$ be a nonsingular potential and assume that $\lim _{\gamma \rightarrow \gamma_{c}}+E_{\gamma}=0$, then

$$
\gamma_{c} \leq \int_{-\infty}^{0} V(x) d x,
$$

Proof.-For $\gamma>\gamma_{c}$ and the normalization $\psi_{E_{\gamma}}(0)=1$

$$
\gamma=\int_{-\infty}^{0}\left(V(x)-E_{\gamma}\right) \psi_{E_{\gamma}}(x) d x .
$$

Taking the limit $\gamma \rightarrow \gamma_{c}{ }^{+}$and using Proposition 4.ii, this gives

$$
\begin{aligned}
\gamma_{c} & =\lim _{\gamma \rightarrow \gamma_{c}} \int_{-\infty}^{0}\left(V(x)-E_{\gamma}\right) \psi_{E_{\gamma}}(x) d x \\
& =\lim _{\gamma \rightarrow \gamma_{c}} \int_{-\infty}^{0} V(x) \psi_{E_{\gamma}}(x) d x \leq \int_{-\infty}^{0} V(x) d x,
\end{aligned}
$$

from where the existence of a negative energy in the spectrum for $\gamma$ satisfying (71) follows.

\section{Energy considerations}

As pointed out in [13], no matter which self-adjoint extension we choose for $\mathcal{H}$, there is always a notion of conserved energy for solutions of the wave equation (15)(16) in the domain $x<0$ of $1+1$ Minkowski spacetime for the solution (17)-(18). If $z \mathcal{H}$ is the chosen self-adjoint extension $(z=D, N, \gamma)$, the energy is defined as

$$
\mathcal{E}_{z}=\frac{1}{2}\left(\left\langle\partial_{t} \phi, \partial_{t} \phi\right\rangle+\left\langle\phi,{ }^{z \mathcal{H}} \phi\right\rangle\right) .
$$

Conservation of $\mathcal{E}_{z}$ follows from $\left[\partial_{t}, z \mathcal{H}\right]=0$ and the selfadjointness of $z \mathcal{H}$,

$$
\begin{aligned}
\dot{\mathcal{E}}_{z} & =\left\langle\partial_{t} \phi, \partial_{t}^{2} \phi\right\rangle+\frac{1}{2}\left(\left\langle\partial_{t} \phi,{ }^{z} \mathcal{H} \phi\right\rangle+\left\langle\phi, \partial_{t}{ }^{z} \mathcal{H} \phi\right\rangle\right) \\
& =\left\langle\partial_{t} \phi, \partial_{t}^{2} \phi+{ }^{z} \mathcal{H} \phi\right\rangle=0 .
\end{aligned}
$$

Integrating (75) by parts, we get [cf. equation (55)]

$$
\begin{aligned}
\mathcal{E}_{z} & =-\left.\frac{1}{2} z \phi^{2}\right|_{x=0}+\frac{1}{2} \int_{-\infty}^{0}\left[\left(\partial_{t} \phi\right)^{2}+\left(\partial_{x} \phi\right)^{2}+V \phi^{2}\right] d x \\
& \equiv \mathcal{E}_{o}-\left.\frac{1}{2} z \phi^{2}\right|_{x=0},
\end{aligned}
$$

where $z=0$ for Dirichlet or Neumann boundary conditions and $z=\gamma=\left.\left(\partial_{x} \phi / \phi\right)\right|_{x=0}$ for Robin boundary conditions. 
The conservation of $\mathcal{E}_{z}$ in (77) can also be derived by applying Gauss's theorem to the conserved current $J_{a}=T_{a b}(\partial / \partial t)^{b}$, where $T_{a b}$ is the energy momentum tensor of the $1+1$ field theory (15)-(16) in the twodimensional (half) Minkowski space. The region of integration is the one bounded by two $t=$ constant surfaces. The integral at the horizon $(x=-\infty)$ vanishes for fields $\phi$ which are square-integrable on $t$ slices, so if we use inertial coordinates $(t, x)$, we get

$0=\int_{-\infty}^{0} T_{t t}(t, x) d x-\int_{-\infty}^{0} T_{t t}\left(t_{o}, x\right) d x-\int_{t_{o}}^{t} T_{t x}\left(t^{\prime}, 0\right) d t^{\prime}$,

where $T_{a b}=\partial_{a} \phi \partial_{b} \phi-\frac{1}{2} \eta_{a b}\left(\partial_{c} \phi \partial^{c} \phi+V \phi^{2}\right)$. Note that

$$
T_{t t}=\frac{1}{2}\left(\dot{\phi}^{2}+\phi^{\prime 2}+V \phi^{2}\right),
$$

and $T_{t x}=\dot{\phi} \phi^{\prime}$, then

$$
\begin{aligned}
\lim _{x \rightarrow 0^{-}} T_{t x} & =\lim _{x \rightarrow 0^{-}} \dot{\phi} \phi^{\prime} \\
& \rightarrow \begin{cases}0, & \mathrm{~N} \text { or D boundary conditions } \\
\frac{1}{2} \gamma \partial_{t}\left(\phi^{2}\right), & \text { Robin boundary conditions. }\end{cases}
\end{aligned}
$$

Thus, for $\mathrm{N}$ or $\mathrm{D}$ boundary conditions the third term in (78) vanishes, there is no flux of energy at the conformal boundary and the canonical energy $\mathcal{E}_{o}$ defined in (77) is conserved. As seen from Eq. (80), the form (56) of the Robin boundary condition is crucial for the existence of the conserved quantity $z=\gamma$ in (77), as it allows the flux of energy at infinity (the timelike boundary) to be integrated, reducing (78) to

$$
\mathcal{E}_{o}(t)-\mathcal{E}_{o}\left(t_{o}\right)=\frac{\gamma}{2}\left[\phi^{2}(t, 0)-\phi^{2}\left(t_{o}, 0\right)\right],
$$

from where the conservation of $\mathcal{E}_{z}$ for the $z=\gamma$ case of (77) follows. Note from (80) and (81) that the change $\gamma \rightarrow-\gamma$ reverses the sign of the energy flow at $x=0$. Note also that a positive $\gamma$ in (81) opens the possibility of an unbounded growth of the canonical energy $\mathcal{E}_{o}$ due to energy pumped in from the boundary [right-hand side in (81)], but the fact that the critical value $\gamma_{c}$ for instability is, in general, strictly positive implies that a flux of energy from the boundary is not a sufficient condition for instability. What happens for $\gamma>\gamma_{c}$ is that the equation of motion allows that $\phi(t, 0)$ grew unbounded together with the energy $\sim \phi^{2}(t, 0)$ pumped into the system, this being the mechanism driving the instability. This can be understood with the help of the toy models of the following section: Eq. (13) can be regarded as transverse oscillations of a semi-infinite string $x<0$ [with elastic $x$-dependent restoring forces when
$V(x)>0]$, and the boundary condition $\phi^{\prime}=\gamma \phi$ at $x=0$ is equivalent to an elastic restoring force at $x=0$ with elastic constant proportional to $-\gamma$. For positive $\gamma$, the force is not restoring but repulsive and proportional to $\phi(t, 0)$ and, if $\gamma>\gamma_{c}$, it allows for solutions with $\phi(t, 0)$ increasing without bound. This negative elastic potential energy is compensated with a diverging positive energy in the string modes [see Eq. (81)].

\section{Toy models}

\section{Vanishing potential: A semi-infinite string}

Consider a string extending along the $x<0$ half axis and oscillating in the $(x, y)$ plane, and let $\phi(x, t)$ be the $y$ displacement at time $t$. For small $\partial_{x} \phi$ we use the standard approximation $\partial_{x} \phi=\tan (\alpha) \simeq \sin (\alpha)$ for the angle $\alpha$ of the string with respect to the horizontal at $x$ and calculate the vertical component of the tension $T$ as $T_{y} \simeq T \partial_{x} \phi$, then Newton's law applied to the piece of string extending from $x$ to $x+\Delta x$ gives

$$
T\left[\partial_{x} \phi(x+\Delta x, t)-\partial_{x} \phi(x, t)\right]=\rho \Delta x \partial_{t}^{2} \phi,
$$

where $\rho$ is the mass per unit length. Taking the limit $\Delta x \rightarrow 0$, we obtain the wave equation

$$
\frac{1}{c^{2}} \partial_{t}^{2} \phi-\partial_{x}^{2} \phi=0, \quad c^{2}=T / \rho,
$$

which, after rescaling $t$, has the form (15)-(16) with $\mathcal{H}=-\partial_{x}^{2}$, that is, $V=0$.

For a string with a right end at $x=0$, Newton's law applied to the $-\Delta x<x<0$ piece of the string,

$$
f-T \partial_{x} \phi(0-\Delta x, t)=\rho \Delta x \partial_{t}^{2} \phi,
$$

gives, after taking the limit $\Delta x \rightarrow 0$, the force $f$ applied to the string end at $x=0$,

$$
f=T \partial_{x} \phi(0, t)
$$

Dirichlet boundary conditions $\phi(0, t)=0$ corresponds to fixing the string at the $x=0$ end, Neumann boundary conditions $\partial_{x} \phi(0, t)=0$ to leaving it free $(f=0)$, and Robin boundary conditions $\partial_{x} \phi(0, t)=\gamma \phi(0, t)$ to subjecting the end of the string to an elastic force

$$
f=\gamma T \phi(0, t),
$$

which corresponds to a spring with elastic constant $k=$ $-\gamma T$ if $\gamma<0$ and gives a "repulsive elastic force" if $\gamma>0$.

Dirichlet boundary conditions: The eigenfunctions of ${ }^{D} \mathcal{H}$ are 


$$
\psi_{\left(E=k^{2}\right)}=\sqrt{\frac{2}{\pi}} \sin (k x), \quad k>0 .
$$

These were normalized such that

$$
\begin{aligned}
\left\langle\psi_{\left(E=k^{2}\right)}, \psi_{\left(E=l^{2}\right)}\right\rangle & =\frac{2}{\pi} \int_{-\infty}^{0} \sin (k x) \sin (l x) d x \\
& =\delta(k-l),(k, l>0) .
\end{aligned}
$$

Functions $\psi$ satisfying the Dirichlet boundary conditions extend naturally to odd functions in $\mathbb{R}$. The completeness of the above set of eigenfunctions then follows from the sine Fourier transform theorem for odd functions as follows:

$$
\psi(x)=\sqrt{\frac{2}{\pi}} \int_{0}^{\infty} A_{k} \sin (k x) d k,
$$

where

$$
A_{k}=\sqrt{\frac{2}{\pi}} \int_{-\infty}^{0} \psi(x) \sin (k x) d x
$$

The conserved energy in this case is $\mathcal{E}_{o}$ defined in (77). Energy can be transferred among the different normal modes of the string but remains conserved. This is due to the fact that the external force that keeps the $x=0$ end of the string fixed does no work on the string.

Neumann boundary conditions: The eigenfunctions of ${ }^{N} \mathcal{H}$ are

$$
\psi_{\left(E=k^{2}\right)}=\sqrt{\frac{2}{\pi}} \cos (k x), \quad k>0 .
$$

These were normalized such that

$$
\begin{aligned}
\left\langle\psi_{\left(E=k^{2}\right)}, \psi_{\left(E=l^{2}\right)}\right\rangle & =\frac{2}{\pi} \int_{-\infty}^{0} \cos (k x) \cos (l x) d x \\
& =\delta(k-l), \quad(k, l>0) .
\end{aligned}
$$

Functions $\psi$ satisfying the Neumann boundary conditions are naturally extended to even functions in $\mathbb{R}$. The completeness of the above set of eigenfunctions then follows from the cosine Fourier transform theorem of even functions.

The conserved energy in this case is again $\mathcal{E}_{o}$ defined in (77). Vibrational energy can be transferred among the different normal modes of the string but remains conserved. This is due to the fact that no external force is acting on the $x=0$ end of the string.

Robin boundary conditions: For any value of $\gamma$, the generalized eigenfunctions corresponding to the continuum spectrum are
$\psi_{\left(E=k^{2}\right)}=\sqrt{\frac{2}{\pi}}\left(1+\frac{k^{2}}{\gamma^{2}}\right)^{-1 / 2}\left[\sin (k x)+\frac{k}{\gamma} \cos (k x)\right]$,

$$
k>0 \text {. }
$$

These were normalized such that

$$
\int_{-\infty}^{0} \psi_{\left(E=k^{2}\right)} \psi_{\left(E=l^{2}\right)} d x=\delta(k-l)
$$

To verify (94), we use the integrals (88) and (92) together with the distributional identity

$$
\int_{-\infty}^{0} \sin (l x) \cos (k x) d x=\frac{l}{(k+l)(k-l)}
$$

From Proposition 5.iv we expect instabilities when $\gamma>0$. This is trivially verified, the only (Proposition 4.iii) bound state of $\gamma \mathcal{H}$ for $\gamma>0$ is

$$
\psi_{\left(E=-\gamma^{2}\right)}=\sqrt{2 \gamma} e^{\gamma x},
$$

where we have normalized such that the integral of $\psi_{\left(E=-\gamma^{2}\right)}^{2}$ equals one. This function has to be added to the set (93) to form a complete orthonormal set for the domain of $\gamma \mathcal{H}$ when $\gamma>0$. To verify that (96) is orthogonal to the functions (93), we use

$$
\int_{-\infty}^{0} e^{\gamma x} e^{-i k x} d x=\frac{1}{\gamma-i k}, \quad \gamma>0
$$

We can also understand the expansion of functions satisfying (56) in terms of ordinary Fourier transforms by means of the following observation: the function $\tau=$ $\psi-\partial_{x} \psi / \gamma$ vanishes at $x=0$, and can naturally be extended to an odd function in $\mathbb{R}$, for which the sine Fourier representation is possible,

$$
\begin{aligned}
\tau(x) & =\sqrt{\frac{2}{\pi}} \int_{0}^{\infty} \tau_{k} \sin (k x) d k, \\
\tau_{k} & =\sqrt{\frac{2}{\pi}} \int_{-\infty}^{0} \tau(x) \sin (k x) d x .
\end{aligned}
$$

$\psi$ can be easily recovered from $\tau$,

$$
\psi(x)=e^{\gamma x}\left[\psi(0)+\gamma \int_{x}^{0} \tau(u) e^{-\gamma u} d u\right] .
$$

If we use the representation (98) of $\tau$ in the above equation, we arrive at the following expansion of $\psi$ in terms of $\psi_{\left(E=-\gamma^{2}\right)}$ and the $\psi_{\left(E=k^{2}\right)}$ above: 


$$
\begin{aligned}
\psi(x)= & {\left[\psi(0)-\sqrt{\frac{2}{\pi}} \int_{0}^{\infty} d k \frac{\gamma k \tau_{k}}{\gamma^{2}+k^{2}}\right] e^{\gamma x} } \\
& +\sqrt{\frac{2}{\pi}} \int_{0}^{\infty} \frac{\gamma^{2} \tau_{k}}{\gamma^{2}+k^{2}}\left(\sin (k x)+\frac{k}{\gamma} \cos (k x)\right) d k .
\end{aligned}
$$

It is a nontrivial fact that the coefficient between square brackets above vanishes if $\gamma<0$.

The conserved energy $\mathcal{E}_{\gamma}$ [Eq. (77)] contains two terms: the string vibrational energy $\mathcal{E}_{o}$ and the potential energy $-\gamma \phi^{2}(x=0, t) / 2$ of the force acting on the $x=0$ end of the string. For negative $\gamma$, this is an ordinary elastic force pulling towards $\phi=0$ : we may imagine that the $x=0$ end of the string is attached to a spring of elastic constant $-\gamma$, then energy flows from the string to the spring and vice versa in such a way that the total energy $\mathcal{E}_{\gamma}$ remains constant. Since the spring potential energy is positive definite, the amount of energy the spring can transfer to the string is finite; this keeps the string vibrations bounded. For positive $\gamma$, instead, the force at the $x=0$ end is "repulsive elastic," pushing away the string end with an intensity that increases as $\phi(x=0)$ grows. The repulsive elastic potential $-\gamma \phi^{2} / 2$ is unbounded from below; it can feed the string with an unlimited amount of energy and produce unbounded oscillations.

The string analogy can be extended to the $V(x) \geq 0$ case by assuming that, besides the string tension, there is an $x$-dependent restoring elastic force pulling the string to the $\phi=0$ configuration. In this case, instead of (82) we have

$$
\begin{aligned}
& T\left[\partial_{x} \phi(x+\Delta x, t)-\partial_{x} \phi(x, t)\right] \\
& \quad-V(x) T \Delta x \phi(x, t)=\rho \Delta x \partial_{t}^{2} \phi,
\end{aligned}
$$

which, taking the limit $\Delta x \rightarrow 0$ in (101) and rescaling $t$, gives (15)-(16).

\section{A step potential}

Consider now the case

$$
V(x)= \begin{cases}0 & x<-a \\ V_{o} & a<x \leq 0,\end{cases}
$$

where $V_{o}$ and $a$ are positive, and assume ${ }^{\gamma} \mathcal{H}$ has a negative energy eigenvalue $E=-\alpha^{2}, \alpha>0$. (Note that a single finite discontinuity in $V$ does not invalidate the results of the previous section.) The wave function is proportional to

$\psi(x)= \begin{cases}\exp (\alpha(x+a)), & x<-a \\ \cosh (\beta(x+a))+\frac{\alpha}{\beta} \sinh (\beta(x+a)), & -a<x \leq 0,\end{cases}$

where

$$
\beta=\sqrt{\alpha^{2}+V_{o}} .
$$

This function is $C^{1}$ and satisfies the properties in Proposition 4. Imposing (56) on (103) gives the following relation:

$$
\gamma=\beta \frac{\beta \tanh (\beta a)+\alpha}{\beta+\alpha \tanh (\beta a)} .
$$

Inserting (104) into (105), we find that $d \gamma / d \alpha>0$ for positive $\alpha$. Since $\gamma \sim \alpha$ for $\alpha \rightarrow \infty$, we conclude that, as $\alpha$ goes from zero to infinity, $\gamma$ ranges from

$$
\gamma_{c}=\sqrt{V_{0}} \tanh \left(\sqrt{V_{0}} a\right)
$$

to infinity. This example is useful because $\gamma_{c}$ introduced in Proposition 5.ii can be explicitly calculated, Eq. (106). If $\gamma>\gamma_{c}$, (105) has a unique solution $\alpha$, which gives a unique bound state, of energy $E=-\alpha^{2}$. On the other hand, if $\gamma \leq \gamma_{c}$, there are no bound states. The bounds (59) and (71) can be easily checked in this example; moreover, the example shows that (71) cannot be improved as for small $a$ (106) gives

$$
\gamma_{c}=a V_{o}+\mathcal{O}\left(a^{3}\right)=\int_{-\infty}^{0} V d x+\mathcal{O}\left(a^{3}\right) .
$$

\section{ROBIN INSTABILITIES IN SAdS}

In Sec. II B 1 we proved that Dirichlet is the only possible choice of boundary condition at infinity for a massless scalar field and that, moreover, the field is stable, in the sense that exponentially growing modes are not allowed. The uniqueness of the dynamics is due to the fact that the mode potentials (31) are LP at $x=0$. For Maxwell fields and gravitational perturbations, the mode potentials are (41) and (44) and (46), respectively, and they are all LC at $x=0$. Besides, these potentials are continuous for $x \in(-\infty, 0]$, satisfy (19) and behave near $x=0$ as in (49). Thus, the results in Sec. III apply to these cases. In particular, instabilities are to be expected for certain Robin boundary conditions. In this section we show that Robin boundary conditions arise naturally for Maxwell fields and gravitational perturbations on $\mathrm{SAdS}_{4}$ and explore the associated "Robin instabilities."

\section{A. Maxwell fields}

In what follows we show how the mode conserved energy resulting from Eqs. (77) and (81) is connected to the electromagnetic field energy. The electromagnetic field modes are labeled by $(p= \pm, \ell, m)$ according to (37) and (38), and (77) and (81) imply that for any function $Q(p, \ell, m)$ 


$$
\begin{aligned}
& \left.\sum_{(\ell, m, p= \pm)} Q(p, \ell, m) \int_{-\infty}^{0}\left[\dot{\phi}_{(p, \ell, m)}^{2}+\phi_{(p, \ell, m)}^{\prime 2}+V_{\ell}^{\mathrm{Max}} \phi_{(p, \ell, m)}^{2}\right] d x\right|_{t} \\
& =\left.\sum_{(\ell, m, p= \pm)} Q(p, \ell, m) \int_{-\infty}^{0}\left[\dot{\phi}_{(p, \ell, m)}^{2}+\phi_{(p, \ell, m)}^{\prime 2}+V_{\ell}^{\mathrm{Max}} \phi_{(p, \ell, m)}^{2}\right] d x\right|_{t_{o}} \\
& \quad+\left.\sum_{(\ell, m, p= \pm)} Q(p, \ell, m) \gamma_{(p, \ell, m)}\left(\phi_{(p, \ell, m)}(s, 0)\right)^{2}\right|_{\substack{s=t \\
s=t_{0}}} .
\end{aligned}
$$

We will show that the choice $Q(p, \ell, m)=\ell(\ell+1) / 2$ in (108) gives the balance equation for the change of electromagnetic energy due to the energy flow from infinity. Energy is measured using the conservation of $J_{\alpha}:=T_{\alpha \beta} \xi^{\beta}$ for $\xi^{\beta} \partial / \partial x^{\beta}=\partial / \partial t$ the timelike Killing vector field and $T_{\alpha \beta}$ the energy momentum tensor of the Maxwell field,

$$
T_{\alpha \beta}=\frac{1}{4 \pi}\left(F_{\alpha \gamma} F_{\beta}^{\gamma}-\frac{1}{4} g_{\alpha \beta} F_{\gamma \delta} F^{\gamma \delta}\right) .
$$

A similar result could be obtained in the gravity case, with $T_{\alpha \beta}$ the effective energy momentum tensor quadratic in the first-order fields that sources the second-order perturbation equations; however, we have found that this calculation becomes unwieldy even using symbolic manipulation computing.

Applying Gauss's theorem to $J^{\alpha}$ in a region $\Omega$ of the spacetime limited by two $t=$ constant surfaces gives

$$
\begin{aligned}
0= & \int_{\Omega} \nabla_{\alpha} J^{\alpha}=\int_{\partial \Omega} J_{\alpha} n^{\alpha}=\int_{\Sigma_{t}} J_{\alpha} n_{\Sigma_{t}}^{\alpha} \\
& +\int_{\Sigma_{t_{0}}} J_{\alpha} n_{\Sigma_{t_{o}}}^{\alpha}+\int_{\mathcal{I}_{t}} J_{\alpha} n_{\mathcal{I}}^{\alpha},
\end{aligned}
$$

where $\mathcal{I}_{t}$ is the $R \rightarrow \infty$ limit of an $r=R$ hypersurface extending from $\Sigma_{t_{o}}$ to $\Sigma_{t}, t>t_{o}$. The induced volume elements on the hypersurfaces are understood on the integrals, the outer pointing unit normal vectors are $n_{\Sigma_{t}}^{\alpha} \partial / \partial x^{\alpha}=f^{-1 / 2} \partial / \partial t, \quad n_{\Sigma_{t}}^{\alpha} \partial / \partial x^{\alpha}=-f^{-1 / 2} \partial / \partial t \quad$ and $n_{\mathcal{I}_{t}}^{\alpha} \partial / \partial x^{\alpha}=f^{1 / 2} \partial / \partial r$. We can therefore rewrite (110) as

$$
\begin{array}{rl}
\int_{-\infty}^{0} & d x \int_{S^{2}} T_{t t}(t, r, \theta, \phi) \sin \theta d \theta d \phi \\
= & \int_{-\infty}^{0} d x \int_{S^{2}} T_{t t}\left(t_{o}, r, \theta, \phi\right) \sin \theta d \theta d \phi \\
& +\lim _{R \rightarrow \infty} R^{2} f(R) \int_{t_{o}}^{t} d t \int_{S^{2}} T_{t r}(t, R, \theta, \phi) \sin (\theta) d \theta d \phi
\end{array}
$$

where the second term on the right-hand side gives the failure for the "standard energy" to be conserved. Inserting
$A_{\beta}=A_{\beta}^{(-)}+A_{\beta}^{(+)}$given in (37)-(38) into $F=d A$ and (109) gives (a prime denotes $\partial_{x}=f \partial_{r}$ )

$$
\begin{aligned}
\left.\left(J_{\alpha}^{(\ell, m)} n_{\mathcal{I}}^{\alpha}\right)\right|_{r=R}= & \left.\sqrt{f} T_{t r}\right|_{r=R}=\frac{1}{4 \pi R^{2} \sqrt{f(R)}} \\
& \times\left[\dot{\phi}_{(\ell, m)}^{+} \phi_{(\ell, m)}^{\prime+}+\dot{\phi}_{(\ell, m)}^{-} \phi_{(\ell, m)}^{\prime}\right] \\
& \times\left[\left(\partial_{\theta} S_{(\ell, m)}\right)^{2}+\frac{1}{\sin ^{2} \theta}\left(\partial_{\phi} S_{(\ell, m)}\right)^{2}\right] .
\end{aligned}
$$

Note that in the $R \rightarrow \infty$ limit, assuming Robin boundary conditions gives the limit $\dot{\phi}_{(p, \ell, m)} \phi_{(p, \ell, m)}^{\prime}=$ $\frac{d}{d t}\left[\gamma_{(p, \ell, m)} \phi_{(p, \ell, m)}^{2} / 2\right]\left[\gamma_{(p, \ell, m)}\right.$ the Robin constant (56) for the mode $(p= \pm, \ell, m)]$. This fact allows us to integrate the flux at $\mathcal{I}_{t}$. Note also that changing the sign of $\gamma_{(p, \ell, m)}$ reverses the direction of the flux at $\mathcal{I}_{t}$.

To proceed we use the fact that

$$
\begin{aligned}
\int_{S^{2}} & {\left[\left(\partial_{\theta} S_{(\ell, m)}\right)^{2}+\frac{1}{\sin ^{2} \theta}\left(\partial_{\phi} S_{(\ell, m)}\right)^{2}\right] \sin (\theta) d \theta d \phi } \\
= & \int_{S^{2}} \hat{D}^{A} S_{(\ell, m)} \hat{D}_{A} S_{(\ell, m)}=-\int_{S^{2}} S_{(\ell, m)} \hat{D}^{A} \hat{D}_{A} S_{(\ell, m)} \\
& =4 \pi \ell(\ell+1),
\end{aligned}
$$

where $\hat{D}_{A}$ is the covariant derivative on $S^{2}$ and we used the orthonormality of the $S_{(\ell, m)}$. After a lengthy calculation, we find that (111) reduces to (108) with $Q(p, \ell, m)=$ $\ell(\ell+1) / 2$, as anticipated.

We now comment briefly on the stability of Maxwell fields. Note that $V_{\ell}^{\operatorname{Max}}$ is nonsingular and non-negative. Thus, the results in Propositions 1-6 apply. In particular: i) the field is stable if either Dirichlet, Neumann or Robin boundary conditions with negative $\gamma_{(p= \pm, \ell, m)}$ are chosen for every $\phi_{(\ell, m)}^{p= \pm}$ (Proposition 3), and ii) the field is unstable if there is a mode $(p, \ell, m)$ for which $\gamma_{(p, \ell, m)}>$ $\sqrt{\ell(\ell+1)} / r_{h}$ (Proposition 1). For the characteristics of the unstable modes the remaining Propositions apply.

Note the rather indirect relation between the boundary conditions on the electromagnetic field $F_{\alpha \beta}$ and those on the mode master variables $\phi_{(\ell, m)}^{ \pm}$: from (37), $F=d A$ with 


$$
A=\sum_{(\ell, m)}\left[\left(\partial_{x} \phi^{(+, \ell, m)} d t+\partial_{t} \phi^{(+, \ell, m)} d x\right) S_{(\ell, m)}+\phi^{(-, \ell, m)}\left(\partial_{\phi} S_{(\ell, m)} / \sin (\theta) d \theta-\sin (\theta) \partial_{\theta} S_{(\ell, m)} d \phi\right)\right] .
$$

As an example, the condition $F(\cdot, \partial / \partial x)=0$ at the boundary implies Dirichlet boundary conditions for the $\phi_{(\ell, m)}^{+}$and Neumann boundary conditions on the $\phi_{(\ell, m)}^{-}$.

\section{B. Linearized gravity}

Let $C_{\alpha \beta \gamma \delta}$ be the Weyl tensor, ${ }^{*} C^{\alpha \beta \gamma \delta}$ its dual. Define the algebraic curvature scalars

$$
\begin{aligned}
& Q_{+}=\frac{1}{48} C^{\alpha \beta \gamma \delta} C_{\alpha \beta \gamma \delta}, \\
& Q_{-}=\frac{1}{48}{ }^{*} C^{\alpha \beta \gamma \delta} C_{\alpha \beta \gamma \delta},
\end{aligned}
$$

and the differential curvature scalar

$$
X=\frac{1}{720}\left(\nabla_{\epsilon} C_{\alpha \beta \gamma \delta}\right)\left(\nabla^{\epsilon} C^{\alpha \beta \gamma \delta}\right) .
$$

For $\mathrm{S}(\mathrm{A}) \mathrm{dS}_{4}$ these fields are

$$
\begin{aligned}
Q_{+}^{\mathrm{SAdS}} & =\frac{M^{2}}{r^{6}}, \quad Q_{-}^{\mathrm{SAdS}}=0, \\
X^{\mathrm{SAdS}} & =\frac{M^{2}}{3 r^{9}}\left(\Lambda r^{3}-3 r+6 M\right) .
\end{aligned}
$$

Consider the first-order perturbation of these fields, $\delta Q_{+}$, $\delta Q_{-}$and $\delta X$. From symmetry arguments one can show that for odd perturbations only $\delta Q_{-} \neq 0$, whereas for even perturbations $\delta Q_{-}=0$, while $\delta Q_{+}$and $\delta X$ are nonzero. The scalar fields

$$
G_{-}=\delta Q_{-}
$$

and

$$
G_{+}=\left(9 M-4 r+\Lambda r^{3}\right) \delta Q_{+}+3 r^{3} \delta X
$$

are gauge-invariant and encode all the gauge-invariant information of the perturbation; in particular, it is possible to reconstruct the metric perturbation in a chosen gauge from the $G_{ \pm}$fields $[1,2]$.

Schwarzschild black hole stability studies prior to [1] were limited to placing pointwise bounds on the ReggeWheeler and Zerilli master fields $\phi_{(\ell, m)}^{ \pm}(t, r)$ (Wald, reference [23]) or analyzing the large $t$ decay of these fields (Price [24], Brady et al. [25]). The $\phi_{(\ell, m)}^{ \pm}$enter the metric perturbation (in, say, the Regge-Wheeler gauge), in a series of the form

$$
h_{\alpha \beta}=\sum_{(\ell, m, p= \pm)} D_{\alpha \beta}^{(\ell, m, p)}\left[\phi_{(\ell, m)}^{(p)}, S_{(\ell, m)}\right]
$$

where the differential operators $D_{\alpha \beta}^{(\ell, m, p)}$ are second-order and linear. The separation of variables in (120) makes the linearized Einstein equations equivalent to the $1+1$ wave equations satisfied by the $\phi_{(\ell, m)}^{ \pm}(t, r)$ and the spherical harmonic equation satisfied by the $S_{(\ell, m)}(\theta, \phi)$. It is clear from (120) that the relation of the $\phi_{(\ell, m)}^{(p)}$ to measurable perturbation effects is remote: four derivatives of these fields enter a single harmonic component of the curvature. Therefore, the boundedness of isolated $\phi_{(\ell, m)}^{ \pm}(t, r)$ fields tells us little about the magnitude of the perturbation.

The nonmodal stability concept introduced in [1] is based on the pointwise boundedness (and decay, see [2]) of the $G_{ \pm}$, which are measurable geometric quantities on the 4D background spacetime that properly record the effect of the perturbation on the geometry. Not only is it established that there is a large $t$ decay of the perturbed black hole to a member of the Kerr-dS family, but also that there are no transient growths of the $G_{ \pm}$, something that modal stability cannot rule out. Examples of modally stable systems for which the isolated modes decay exponentially with $t$ and yet measurable quantities experience large transient growths are seen, e.g., in wall-bounded shear flows (see, e.g., [26]).

By iterating the linearized Einstein equations we arrive, after some work, to a relatively simple on-shell form of $G_{ \pm}$. For $G_{-}$we find

$$
\begin{aligned}
G_{-}= & -\frac{6 M}{r^{7}} \sqrt{\frac{4 \pi}{3}} \sum_{m=1}^{3} j^{(m)} S_{(\ell=1, m)} \\
& -\frac{3 M}{r^{5}} \sum_{\ell>1, m} \frac{(\ell+2) !}{(\ell-2) !} \frac{\phi_{(\ell, m)}^{-}}{r} S_{(\ell, m)},
\end{aligned}
$$

where the first term contains the $\ell=1$ static contribution (not considered in the previous sections), $j^{(m)}, m=1,2,3$ being the components of the perturbed black hole angular momentum. In view of (121), $r^{5} G_{-}$satisfies the fourdimensional Regge-Wheeler equation (4). For the static $\ell=1$ in (121) term this can be checked by a direct calculation; for the $\ell>1$ series this follows from the spherical harmonic equation and the form of the $V_{\ell}^{(-)}$ potentials that enter the $1+1$ wave equation satisfied by $\phi_{(\ell, m)}^{-}$, which contains the required $\ell(\ell+1) / r^{2}$ term, see (44). 
Due to the intricate $\ell$ dependence of the $V_{\ell}^{(+)}$in (46), no similar construction leading to a four-dimensional wave equation can be made using the Zerilli fields $\phi_{(\ell, m)}^{+}$. These fields enter the on-shell expression of $G_{+}$as follows:

$G_{+}=-\frac{2 M \delta M}{r^{5}}+\frac{M}{2 r^{4}} \sum_{\ell \geq 2} \frac{(\ell+2) !}{(\ell-2) !}\left[f \partial_{r}+Z_{\ell}\right] \phi_{(\ell, m)}^{+} S_{(\ell, m)}$,

where $\delta M$ is the mass variation that comes from the $\ell=0$ even perturbation (which is time-independent and was not considered in the previous sections), and

$$
\begin{aligned}
Z_{\ell} & =\frac{2 M \Lambda r^{3}+\mu r(r-3 M)-6 M^{2}}{r^{2}(\mu r+6 M)}, \\
\mu & =(\ell-1)(\ell+2) .
\end{aligned}
$$

To see how natural imposing Robin boundary conditions on the $\phi_{(\ell, m)}^{ \pm}$is, note that Dirichlet conditions on the $G_{ \pm}$are equivalent to mixed Dirichlet/Robin conditions on the $\phi_{(\ell, m)}^{ \pm}$,

$\left.\phi_{(\ell, m)}^{-}\right|_{x=0}=0,\left.\quad \frac{\partial_{x} \phi_{(\ell, m)}^{+}}{\phi_{(\ell, m)}^{+}}\right|_{x=0}=-\frac{2 M \Lambda}{(\ell-1)(\ell+2)}$,

whereas imposing Neumann or Robin boundary conditions on the $G_{ \pm}$gives Robin conditions on the $\phi_{(\ell, m)}^{ \pm}$. The suitability of a boundary condition depends on the problem at hand, and for linear stability studies it should be kept in mind that the fields $\phi_{(\ell, m)}^{ \pm}$, although convenient to disentangle the linearized Einstein equations, are not relevant since they are not directly measurable quantities.

Another context where Robin boundary conditions on the $\phi_{(\ell, m)}^{ \pm}$arise is that of the AdS/CFT correspondence, under which the unperturbed background corresponds to a perfect fluid in the boundary and one is interested in metric perturbations that, in a preferred gauge, vanish in large $r=$ constant surfaces $[27,28]$.

The metric for the CFT is conformally related to that induced on large $r$ surfaces by

$$
d s_{\infty}^{2}=\lim _{r \rightarrow \infty}\left(-\frac{3}{\Lambda r^{2}} d s_{r}^{2}\right) .
$$

In the unperturbed background $d s_{r}^{2}$ is obtained by setting $d r=0$ in (1)-(2), and the above limit gives

$$
d s_{\infty}^{2}=-d t^{2}-\frac{3}{\Lambda}\left(d \theta^{2}+\sin ^{2}(\theta) d \phi^{2}\right) .
$$

For even perturbations in the Regge-Wheeler gauge, (125) gives [see equations (3.46) in [27] and (129) in [2], where $\mathcal{J}$ is defined] $d s_{\infty}^{2}=-d t^{2}-\frac{3}{\Lambda}\left(1+\frac{1}{2} \lim _{r \rightarrow \infty} \mathcal{J}\right)\left(d \theta^{2}+\sin ^{2}(\theta) d \phi^{2}\right)$.

Thus, for the induced metric not to be perturbed we require that the $(\ell, m)$ harmonic component $\mathcal{J}_{(\ell, m)}$ of $\mathcal{J}$ vanishes for large $r$. From equations (151), (153) and (154) in [2] we find that

$$
\mathcal{J}_{(\ell, m)} \propto 2 f(r) \partial_{r} \zeta_{(\ell, m)}^{+}+\frac{\ell(\ell+1)}{r} \zeta_{(\ell, m)}^{+},
$$

where

$$
\zeta_{(\ell, m)}^{+}=\left((\ell+2)(\ell-1)+\frac{6 M}{r}\right) \phi_{(\ell, m)}^{+} .
$$

Keeping the conformal boundary metric unperturbed will then impose the following Robin boundary condition for $\phi_{(\ell, m)}^{+}$at $x=0$ :

$$
\partial_{x} \phi_{(\ell, m)}^{+}=-\frac{2 M \Lambda}{(\ell+2)(\ell-1)} \phi_{(\ell, m)}^{+} .
$$

\section{Explicit unstable modes}

The following field was reported in [2] as an unstable solution for the even gravitational perturbations of $\mathrm{SAdS}_{4}$ [equations (15)-(16) with potential (46)] satisfying Robin boundary conditions:

$$
\phi_{(\ell, m)}^{+u n s t}=\chi_{\ell}^{+}(r) \exp \left(w_{\ell} t\right)
$$

where

$$
\chi_{\ell}^{+}(r)=\frac{r \exp \left(w_{\ell} x\right)}{(\ell+2)(\ell-1) r+6 M},
$$

$x$ is the radial coordinate defined in (5) and

$$
w_{\ell}=\frac{1}{12 M} \frac{(\ell+2) !}{(\ell-2) !} .
$$

$\chi_{\ell}^{+}(r)$ defined in (132) satisfies $\mathcal{H}^{+} \chi_{\ell}^{+}=-w_{\ell}^{2} \chi_{\ell}^{+}$where $\mathcal{H}_{\ell}^{+}$ is the Hamiltonian for even/scalar gravitational perturbations. This equation is satisfied for any value of $M$ and $\Lambda$, as long as $x(r)$ in (132) satisfies $d x / d r=1 / f$ [cf. Eq. (5)] with the appropriate parameters. For $\Lambda=0$, this solution was found by Chandrasekhar [3] when looking for algebraically special perturbations: those with the property where the first-order variation of one of the Weyl scalars $\Psi_{0}$ or $\Psi_{4}$ vanishes. A linearly independent solution with the same negative energy is [3] 


$$
r_{o} \tau_{\ell}^{+}(r)=\chi_{\ell}^{+}(r) \int_{r_{o}}^{r} \frac{d r^{\prime}}{f\left(r^{\prime}\right)\left(\chi_{\ell}^{+}\left(r^{\prime}\right)\right)^{2}} .
$$

Note that changing $r_{o}$ above adds a term proportional to $\chi_{\ell}^{+}(r)$.

Two linearly independent solutions of $\mathcal{H}_{\ell}^{-} \psi_{\ell}^{-}=-w_{\ell}^{2} \psi_{\ell}^{-}$ for the odd (vector) Hamiltonian with the same negative energy $E=-w_{\ell}^{2}$ are [3]

$\chi_{\bar{\ell}}^{-}(r)=\frac{1}{\chi_{\ell}^{+}(r)}, \quad r_{o} \tau_{\ell}^{-}(r)=\chi_{\bar{\ell}}^{-}(r) \int_{r_{o}}^{r} \frac{d r^{\prime}}{f\left(r^{\prime}\right)\left(\chi_{\ell}^{-}\left(r^{\prime}\right)\right)^{2}}$.

The fact that, for $\Lambda \geq 0, x$ ranges from minus infinity as $r \rightarrow r_{h}{ }^{+}$to infinity as $r \rightarrow \infty(\Lambda=0)$ or approaches the cosmological horizon $(\Lambda>0)$, makes the algebraically special perturbations (132), (134) and (135) uninteresting in these cases because all these solutions diverge at least one of these two limits, and so are irrelevant as they are not eigenfunctions of $\mathcal{H}^{ \pm}$. The situation is different for $\Lambda<0$ and also for the nonglobally hyperbolic Schwarzschild naked singularity $(\Lambda=0, M<0)$. For the latter, $x$ can be chosen to range from $x=0$ (the timelike boundary at the $r=0$ singularity) to infinity (as $r \rightarrow \infty$ ) and, as $w_{\ell}<0$ in this case, (132) behaves properly in both limits [this happens only for even perturbations; neither $\chi^{-}(r)$ nor $\tau_{r_{o}}^{-}(r)$ for any $r_{o}$ behave properly]. Moreover, it was found in [29] (see also [30]) that there is a single boundary condition at the $r=0$ timelike boundary that leads to a consistent linear perturbation treatment; therefore, the dynamics are not ambiguous in spite of the nonglobally hyperbolic character of the spacetime. This particular Robin boundary condition is precisely the one satisfied by the mode (132). Since this mode grows exponentially in time, the claim that the Schwarzschild naked singularity is unstable is free of ambiguities [30,31].

In what follows we concentrate on the $\Lambda<0, M>0$ $\mathrm{SAdS}_{4}$ black hole, for which $x \in(-\infty, 0)$, and one can check using (6) that $\chi^{+}(r)$ and

$$
\tau_{\ell}^{-} \equiv{ }_{r_{h}} \tau_{\ell}^{-}(r)=\chi_{\ell}^{-}(r) \int_{r_{h}}^{r} \frac{d r^{\prime}}{f\left(r^{\prime}\right)\left(\chi_{\ell}^{-}\left(r^{\prime}\right)\right)^{2}}
$$

satisfy the bound state, negative energy requirements (52) and (53) for $\mathcal{H}_{(\ell, m)}^{+}$and $\mathcal{H}_{(\ell, m)}^{-}$, respectively, the choice $r_{o}=r_{h}$ above being crucial for this to hold.

The unstable even solution (131) satisfies a Robin boundary condition (56) at $x=0$ with $\gamma$ equal to

$$
\gamma_{\mathrm{Ch}} \equiv w_{\ell}-\frac{2 M \Lambda}{(\ell-1)(\ell+2)}=\frac{\Lambda r_{h}\left(\Lambda r_{h}{ }^{2}-3\right)}{3(\ell-1)(\ell+2)}-\frac{(\ell+2)(\ell+1) \ell(\ell-1)}{2 r_{h}\left(\Lambda r_{h}{ }^{2}-3\right)}
$$

The perturbation of the metric is

$$
h_{(\ell, m)}^{+ \text {unst }}=\exp \left(w_{\ell} v\right) S_{(\ell, m)}\left[\frac{w_{\ell}}{6 M}(r \ell(\ell+1)-6 M) d v \otimes d v+\frac{\ell(\ell+1)}{6 M} r^{2}\left(d \theta \otimes d \theta+\sin ^{2}(\theta) d \phi \otimes d \phi\right)\right],
$$

where we defined $v=t+x$. The above expression shows that the perturbation is well behaved across the future event horizon, defined by $r=r_{h}, v \in \mathbb{R}$. This perturbation has the property of splitting only one of the two pairs of principal null directions of the background so that the perturbed spacetime is Petrov type-II. For generic perturbations, instead, both pairs of principal null directions are split, leaving a type-I spacetime (see [32] for details).

For the pure mode (131), (122) gives

$$
\begin{aligned}
G_{+}\left[\phi_{(\ell, m)}^{+ \text {unst }}\right] & =\frac{(\ell+2) !}{(\ell-2) !}\left(\frac{\ell(\ell+1) r-6 M}{24 r^{5}}\right) \exp \left(w_{\ell} v\right), \\
v & =t+x(r) .
\end{aligned}
$$

The existence of the bound state $\chi_{\ell}^{+}$for the Zerilli SAdS 4 Hamiltonian (15)-(16), which has a positive nonsingular potential (46), implies, in view of Proposition 5.ii, that for even $\ell$ modes the critical value of $\gamma$ for instabilities satisfies

$$
\gamma_{c} \leq \gamma_{\mathrm{Ch}} \simeq \begin{cases}\frac{(\ell+2) !}{(\ell-2) ! \frac{1}{6 r_{h}},}, & r_{h} \rightarrow 0 \\ \frac{\Lambda^{2} r_{h}{ }^{3}}{3(\ell-1)(\ell+2)}, & r_{h} \rightarrow \infty\end{cases}
$$

We can use this to test the upper bound (74) in these limits using (48),

$$
\gamma_{c}<\int_{-\infty}^{0} V_{\ell}^{(+)} d x \simeq \begin{cases}\frac{2 \ell^{2}+2 \ell-3}{2 r_{h}}, & r_{h} \rightarrow 0 \\ \frac{2 \Lambda^{2} r_{h}{ }^{3}}{3(\ell+2)(\ell-1)}, & r_{h} \rightarrow \infty .\end{cases}
$$

For large horizon radii, $\gamma_{\mathrm{Ch}}$ is half the value of the integrated potential, whereas for small $r_{h}$ we find that $\gamma_{\mathrm{Ch}}$ is less than the integrated potential only for $\ell=2$ (the minimum possible $\ell$ value), and grows as $\ell^{4}$ for large $\ell$, whereas the integrated potential grows only as $\ell^{2}$. We should keep in mind, however, that statement (74) cannot be improved, as the $a \rightarrow 0$ limit of the step potential example in Sec. III D 2 saturates this inequality. 


\section{Boundary conditions and even/odd duality breaking}

Unlike the negative mass Schwarzschild solution, for which a unique boundary condition at the conformal timelike boundary is singled out from the infinite set of $z$ conditions $(z=\mathrm{D}, \mathrm{N}$ or $\gamma)$ by a consistency requirement of the linear perturbation scheme [29], for $\mathrm{SAdS}_{4}$ we may choose among Dirichlet, Neumann or Robin boundary conditions with a specific $\gamma_{(p= \pm, \ell, m)}$ for every mode. We recall that the potentials for even perturbations are nonsingular and positive definite, so Propositions 1 to 6 apply to them, whereas odd perturbations have nonsingular potentials, negative near the horizon for small enough $r_{h} / M$, so only Propositions $1-3$ apply to them in general.

If, for every $(p= \pm, \ell, m)$ we choose the $\left.\gamma_{(p= \pm, \ell, m)}\right)$ below the critical value, the resulting dynamics will be stable. However, a single mode for which $\gamma$ is high enough would introduce an instability. This implies that any gravitational stability claim for $\mathrm{SAdS}_{4}$ is meaningful only after specifying the chosen boundary conditions.

In what follows, we proceed to analyze the relation between boundary conditions at the conformal boundary $r=\infty$ and the formal duality exchanging odd and even modes, discovered by Chandrasekhar about thirty years ago $[3,33]$. This duality played a crucial role in the nonmodal stability proof for Schwarzschild black holes when $\Lambda \geq 0$ $[1,2]$ as it allows us to replace all $\phi_{(\ell, m)}^{+}$Zerilli fields with $\phi_{(\ell, m)}^{-}$Regge-Wheeler fields, and then use the boundedness and decay properties of the four-dimensional ReggeWheeler equation (4) for non-negative $\Lambda$. This substitution generically fails when $\Lambda<0$, holding only when related boundary conditions are chosen in the odd and even sectors. These issues are explored in this section.

All the relations we need follow from the observations in $[3,33]$ that

$$
\mathcal{H}_{\ell}^{ \pm}=\mathcal{D}_{\ell}^{ \pm} \mathcal{D}_{\ell}^{\mp}-w_{\ell}^{2},
$$

where

$$
\mathcal{D}_{\ell}^{ \pm}= \pm \partial_{x}+W_{\ell}, \quad W_{\ell}=w_{\ell}+\frac{6 M f}{r(r \mu+6 M)},
$$

and also that

$$
W_{\ell}=\frac{\partial_{x} \chi_{\ell}^{+}}{\chi_{\ell}^{+}}=-\frac{\partial_{x} \chi_{\ell}^{-}}{\chi_{\ell}^{-}},
$$

which can be verified using (132) and (135). From these we find that

$$
\begin{gathered}
V_{\ell}^{+}=W_{\ell}^{\prime}+\left(W_{\ell}\right)^{2}-w_{\ell}^{2}=\frac{\chi_{\ell}^{+\prime \prime}}{\chi_{\ell}^{+}}-w_{\ell}^{2}, \\
V_{\ell}^{-}=-W_{\ell}^{\prime}+\left(W_{\ell}\right)^{2}-w_{\ell}^{2}=\frac{\chi_{\ell}^{-\prime \prime}}{\chi_{\ell}^{-}}-w_{\ell}^{2},
\end{gathered}
$$

where a prime means derivative with respect to $x$. The second form in (145) allows us to write the ordinary differential equation $\mathcal{H}_{\ell}^{+} \psi=E \psi$ as

$$
-\frac{\psi^{\prime \prime}}{\psi}+\frac{\chi_{\ell}^{+\prime \prime}}{\chi_{\ell}^{+}}=\left(E+w_{\ell}^{2}\right)
$$

from where, for $E=-w_{\ell}^{2}$, we readily obtain the two linearly independent solutions $\psi=\chi_{\ell}^{+}$and $\psi=\tau_{\ell}^{+}$given in (134). A similar analysis leads to the unstable odd modes (135).

In view of (142), acting with $\mathcal{D}_{\ell}^{-}$on a solution to the differential equation $\mathcal{H}_{\ell}^{+} \psi^{+}=E \psi^{+}$gives a-possibly trivial-solution $\psi^{-}=\mathcal{D}_{\ell}^{-} \psi^{+}$of $\mathcal{H}_{\ell}^{-} \psi^{-}=E \psi^{-}$and vice versa,

$$
\begin{aligned}
& \mathcal{H}_{\ell}^{+} \psi^{+}=E \psi^{+} \Rightarrow \mathcal{H}_{\ell}^{-}\left(\mathcal{D}_{\ell}^{-} \psi^{+}\right)=E\left(\mathcal{D}_{\ell}^{-} \psi^{+}\right), \\
& \mathcal{H}_{\ell}^{-} \psi^{-}=E \psi^{-} \Rightarrow \mathcal{H}_{\ell}^{+}\left(\mathcal{D}_{\ell}^{+} \psi^{-}\right)=E\left(\mathcal{D}_{\ell}^{+} \psi^{-}\right) .
\end{aligned}
$$

From the equations above we find some trivial cases,

$$
\mathcal{D}_{\ell}^{-} \chi_{\ell}^{+}=0, \quad \mathcal{D}_{\ell}^{+} \chi_{\ell}^{-}=0 .
$$

Note that the most general solution of the equation $\mathcal{D}_{\ell}^{-} \psi^{+}=0\left(\mathcal{D}_{\ell}^{+} \psi^{-}=0\right)$ is a constant times $\chi_{\ell}^{+}\left(\chi_{\ell}^{-}\right)$. For eigenfunctions with eigenvalues different from $-w_{\ell}^{2}$ the effect of $\mathcal{D}_{\ell}^{ \pm}$can be reversed by $\mathcal{D}_{\ell}^{\mp}$ (times a function of $E$ ), and vice versa,

$$
\begin{aligned}
\mathcal{H}_{\ell}^{+} \psi^{+} & =E \psi^{+} \Rightarrow \mathcal{D}_{\ell}^{+}\left(\mathcal{D}_{\ell}^{-} \psi^{+}\right)=\left(\mathcal{H}_{\ell}^{+}+w_{\ell}^{2}\right) \psi^{+} \\
& =\left(E+w_{\ell}^{2}\right) \psi^{+} \neq 0 \\
\mathcal{H}_{\ell}^{-} \psi^{-} & =E \psi^{-} \Rightarrow \mathcal{D}_{\ell}^{-}\left(\mathcal{D}_{\ell}^{+} \psi^{-}\right)=\left(\mathcal{H}_{\ell}^{-}+w_{\ell}^{2}\right) \psi^{-} \\
& =\left(E+w_{\ell}^{2}\right) \psi^{-} \neq 0 .
\end{aligned}
$$

From equations (151) and (152) it follows that if $\psi_{j}^{-}, j=1$, 2, are two linearly independent solutions of $\mathcal{H}_{\ell}^{-} \psi_{j}^{-}=E \psi_{j}^{-}$ with $E \neq-w_{\ell}^{2}$, then $\psi_{j}^{+}=\mathcal{D}_{\ell}^{+} \psi_{j}^{-}, j=1,2$, are two linearly independent solutions of $\mathcal{H}_{\ell}^{+} \psi_{j}^{+}=E \psi_{j}^{+}$, and similarly if we exchange - and + .

We also note from the above equations that

$$
\mathcal{D}_{\ell}^{-} r_{o} \tau_{\ell}^{+}=\chi_{\ell}^{-}, \quad \mathcal{D}_{\ell}^{+} r_{o} \tau_{\ell}^{-}=\chi_{\ell}^{+}
$$

for any $r_{o}$, and that

$$
\mathcal{D}_{\ell}^{+} \kappa_{\ell}^{-}=\chi_{\ell}^{+} \Rightarrow \kappa_{\ell}^{-}=\tau_{\ell}^{-}+\alpha \chi_{\ell}^{-},
$$

where $\tau_{\ell}^{-}$was defined in (136) and $\alpha$ is a constant.

The possibility of exchanging even and odd modes using $\mathcal{D}_{\ell}^{ \pm}$is the duality, peculiar to four dimensions, that we will analyze for $\Lambda<0$ in the remainder of this section. For 
$\Lambda \geq 0$, the fields $\phi_{(\ell, m)}^{ \pm}$belong to $L^{2}(\mathbb{R}, d x)$ and the operators $\mathcal{D}_{\ell}^{ \pm}$give a bijection between the sets of solutions of the odd and even $1+1$ wave equations (see Section IV.5 in [2].) The case where $\Lambda<0$ is much subtler. The linear gravity potentials $V_{\ell}^{ \pm}$are nonsingular, the values at $x=0$ of solutions of $\mathcal{H}_{\ell}^{ \pm} \psi^{ \pm}=E \psi^{ \pm}$and their $x$ derivatives are well defined and generically nonzero [Eq. (50)], so Dirichlet, Neumann or Robin boundary conditions are allowed. Suppose that, for a given $(\ell, m)$, we choose

$$
\left.\psi^{+\prime}\right|_{x=0}=\left.\gamma_{e} \psi^{+}\right|_{x=0}
$$

where, in what follows $\gamma_{e} \in \mathbb{R} \cup\{\infty\}$ to include the cases $\gamma_{e}=0$ (Neumann) and $\gamma_{e}=\infty$ (Dirichlet), and we similarly introduce $\gamma_{o}$ for the odd modes, dropping the $(\ell, m)$ indices for simplicity.

From (143) we find that

$\left.\left(\mathcal{D}_{\ell}^{-} \psi^{+}\right)\right|_{x=0}=-\left.\psi^{+\prime}\right|_{x=0}+\left.\left(W_{\ell} \psi^{+}\right)\right|_{x=0}=\left.\left(W_{\ell}-\gamma_{e}\right) \psi^{+}\right|_{x=0}$

and that

$\left.\left(\mathcal{D}_{\ell}^{-} \psi^{+}\right)^{\prime}\right|_{x=0}=-\left.\psi^{+\prime \prime}\right|_{x=0}+\left.\left(W_{\ell}^{\prime}+\gamma_{e} W_{\ell}\right) \psi^{+}\right|_{x=0}$,

where, from (144),

$$
\left.W\right|_{x=0}=\gamma_{\mathrm{Ch}} \quad \text { and }\left.\quad W^{\prime}\right|_{x=0}=\left(\frac{2 M \Lambda}{\mu}\right)^{2},
$$

and $\gamma_{\mathrm{Ch}}$ was defined in (137). It is easy to prove from these two equations that, in general, there is no function $\gamma_{o}\left(\gamma_{e}\right)$, $\gamma_{e}, \gamma_{o} \in \mathbb{R} \cup\{\infty\}$, such that $\psi^{+1} / \psi^{+}=\gamma_{e}$ at $x=0$ implies $\left(\mathcal{D}_{\ell}^{-} \psi^{+}\right)^{\prime} / \mathcal{D}_{\ell}^{-} \psi^{+}=\gamma_{o}\left(\gamma_{e}\right)$ at $x=0$. To show this, we use the fact that $\psi^{+}$fields satisfying (155) can be expanded using the complete basis of generalized eigenfunctions $\gamma^{e} \psi_{E}^{+}$(we suppress the $\ell$ index) of the corresponding selfadjoint extension $\gamma_{e} \mathcal{H}_{\ell}^{+}$, so that

$$
\psi^{+}=\int d E c_{E}^{\gamma_{e}} \psi_{E}^{+}
$$

where the integral notation includes a sum over bound states, if there were any.

For an energy eigenstate we find from (157) that

$$
\begin{aligned}
\left.\left(\mathcal{D}_{\ell}^{-\gamma_{e}} \psi_{E}^{+}\right)^{\prime}\right|_{x=0} & =\left.\left(\mathcal{H}_{\ell}^{+}-V_{\ell}^{+}+W_{\ell}^{\prime}+\gamma_{e} W_{\ell}\right)^{\gamma_{e}} \psi_{E}^{+}\right|_{x=0} \\
& =\left.\left(E+w_{\ell}^{2}-W_{\ell}^{2}+\gamma_{e} W_{\ell}\right)^{\gamma_{e}} \psi_{E}^{+}\right|_{x=0}
\end{aligned}
$$

which, together with (155), gives

$$
\left.\frac{\left(\mathcal{D}_{\ell}^{-\gamma_{e}} \psi_{E}^{+}\right)^{\prime}}{\mathcal{D}_{\ell}^{-\gamma_{e}} \psi_{E}^{+}}\right|_{x=0}=\left.\frac{E+w_{\ell}^{2}-W_{\ell}^{2}+\gamma_{e} W_{\ell}}{W_{\ell}-\gamma_{e}}\right|_{x=0} .
$$

Since, generically, the quotient above depends on $E$, $\psi^{+1} / \psi^{+}$in (159) will have different values for different functions in the linear space obtained by applying $\mathcal{D}_{\ell}^{-}$to the domain of $\gamma_{e} \mathcal{H}_{\ell}^{+}$, then the dynamics will not be defined in this space since it is not a self-adjoint domain of $\mathcal{H}_{\bar{l}}^{-}$ (note that this problem cannot be fixed by the alternative definitions $\tilde{\mathcal{D}}^{-} \equiv f(E) \mathcal{D}^{-}$of the dual map used in [27,33]). The only exceptions [i.e., situations where the right hand side of (162) does not depend on $E$ ] are: i) when we choose Dirichlet boundary conditions in the even sector, that is, $\gamma_{e} \rightarrow \infty$ in (162), which gives Robin conditions in the odd sector with $\gamma_{o}=-\left.W\right|_{x=0}=-\gamma_{\mathrm{Ch}}$, that is,

$$
\gamma_{o}=-\gamma_{\mathrm{Ch}} \quad\left(\gamma_{e}=\infty\right),
$$

and ii) when we choose Dirichlet boundary conditions in the odd sector, that is, $\gamma_{e}=\left.W\right|_{x=0}=\gamma_{\mathrm{Ch}}$ in (162),

$$
\gamma_{e}=\gamma_{\mathrm{Ch}} \quad\left(\gamma_{o}=\infty\right) \text {. }
$$

The following proposition gives more details about the supersymmetry and these two cases:

Proposition 7. Consider the maps $\mathcal{D}^{ \pm}$defined in (143). In what follows we use the symbol $\gamma \mathcal{H}_{\ell}^{ \pm}$both for the selfadjoint operator and its domain.

(i) The spectra of ${ }^{D} \mathcal{H}_{\ell}^{+}$and ${ }^{-\gamma_{\mathrm{Ch}}} \mathcal{H}_{\ell}^{-}$are non-negative. $\mathcal{D}_{\ell}^{-}:{ }^{D} \mathcal{H}_{\ell}^{+} \rightarrow^{-\gamma_{\mathrm{Cn}}} \mathcal{H}_{\ell}^{-}$is a bijection.

(ii) The spectrum of ${ }^{D} \mathcal{H}_{\ell}^{-}$is non-negative and that of $\gamma_{\mathrm{Cn}} \mathcal{H}_{\ell}^{+}$contains a negative energy with eigenfunction $\chi^{+}$. The map $\mathcal{D}_{\ell}^{-}: \gamma_{\mathrm{Cn}} \mathcal{H}_{\ell}^{+} \rightarrow{ }^{D} \mathcal{H}_{\ell}^{-}$is surjective and has kernel the linear space generated by $\chi_{\ell}^{+}$. The map is surjective and its kernel is the linear space generated by $\mathcal{D}_{\ell}^{+}:{ }^{D} \mathcal{H}_{\ell}^{-} \rightarrow{ }^{\gamma_{\mathrm{Ch}}} \mathcal{H}_{\ell}^{+}$is injective.

(iii) There are no other values of $\gamma, \gamma^{\prime} \in \mathbb{R} \cup\{\infty\}$ such that $\mathcal{D}_{\ell}^{\mp}\left(\gamma^{\prime} \mathcal{H}_{\ell}^{ \pm}\right) \subset \gamma^{\prime} \mathcal{H}_{\ell}^{\mp}$.

Proof.-We have already proven iii).

To prove i) note that the only solution of $\mathcal{D}_{\ell}^{-} \psi^{+}=0$ is a constant times $\chi^{+}$, which does not belong to ${ }^{D} \mathcal{H}_{\ell}^{+}$, so the map in i) has a trivial kernel and therefore is injective. Similarly, the only solution of $\mathcal{D}_{\ell}^{+} \psi^{-}=0$ is a constant times $\chi^{-}=1 / \chi^{+}$which, although satisfies a Robin condition with $\gamma=-\gamma_{\mathrm{Ch}}$ at $x=0$, diverges as $x \rightarrow-\infty$ and so does not belong to ${ }^{-\gamma_{\mathrm{Cn}} \mathcal{H}_{\ell}^{-}}$. This implies that the map $\mathcal{D}_{\ell}^{+}:{ }^{-\gamma_{\mathrm{Cn}}} \mathcal{H}_{\ell}^{-} \rightarrow{ }^{D} \mathcal{H}_{\ell}^{+}$is injective. Since $V_{\ell}^{+}$is non-negative, Proposition 3 applies and the spectrum of ${ }^{D} \mathcal{H}_{\ell}^{+}$is non-negative. The spectrum of ${ }^{-\gamma_{\mathrm{Cn}}} \mathcal{H}_{\ell}^{-}$must also be nonnegative; otherwise, a negative eigenfunction of ${ }^{-\gamma_{\mathrm{Ch}}} \mathcal{H}_{\ell}^{-}$ would be sent by the injective map $\mathcal{D}_{\ell}^{+}$to a negative eigenfunction of ${ }^{D} \mathcal{H}_{\ell}^{+}$, which is a contradiction. This proves that both ${ }^{D} \mathcal{H}_{\ell}^{+}$and ${ }^{-\gamma_{\mathrm{Ch}}} \mathcal{H}_{\ell}^{-}$have non-negative spectra 
and therefore lead to stable dynamics in the even and odd sectors, respectively. To prove that $\mathcal{D}_{\ell}^{-}:{ }^{D} \mathcal{H}_{\ell}^{+} \rightarrow{ }^{-\gamma_{\mathrm{Ch}}} \mathcal{H}_{\ell}^{-}$is onto we proceed as in Lemma 7 in [2]: let $\psi^{-}$be an arbitrary function in ${ }^{-\gamma_{\mathrm{Cn}}} \mathcal{H}_{\ell}^{-}$and $\psi^{-}=\int d E q(E)^{-\gamma_{\mathrm{Ch}}} \psi_{E}^{-}$its expansion in eigenfunctions ${ }^{-\gamma_{\mathrm{Ch}}} \psi_{E}^{-}$of ${ }^{-\gamma_{\mathrm{Ch}}} \mathcal{H}_{\ell}^{-}$. Since the spectrum of ${ }^{-\gamma_{\mathrm{Cn}}} \mathcal{H}_{\ell}^{-}$is non-negative,

$$
\tilde{\psi}^{-}=\int d E\left(\frac{q(E)}{w_{\ell}^{2}+E}\right)^{-\gamma_{\mathrm{Ch}}} \psi_{E}^{-}
$$

is well defined and belongs to ${ }^{-\gamma_{\mathrm{Ch}}} \mathcal{H}_{\ell}^{-}$. Then $\mathcal{D}_{\ell}^{+} \tilde{\psi}^{-}$is in ${ }^{D} \mathcal{H}_{\ell}^{+}$and is the function sent to $\psi^{-}$by $\mathcal{D}_{\ell}^{-}$, as the following calculation shows:

$\mathcal{D}_{\ell}^{-}\left(\mathcal{D}_{\ell}^{+} \tilde{\psi}^{-}\right)=\left(\mathcal{H}_{\ell}^{-}+w_{\ell}^{2}\right) \int d E\left(\frac{q(E)}{w_{\ell}^{2}+E}\right)^{-\gamma_{\mathrm{Ch}}} \psi_{E}^{-}=\psi^{-}$.

This completes the proof of i).

To prove ii) recall that the only solution of $\mathcal{D}_{\ell}^{+} \psi^{-}=0$ is a constant times $\chi^{-}$, which does not belong to ${ }^{D} \mathcal{H}_{\ell}^{-}$; therefore, $\mathcal{D}_{\ell}^{+}:{ }^{D} \mathcal{H}_{\ell}^{-} \rightarrow{ }^{\gamma_{\mathrm{Cn}}} \mathcal{H}_{\ell}^{+}$is injective. We have already proven that the kernel of $\mathcal{D}_{\ell}^{-}: \gamma_{\mathrm{Cn}} \mathcal{H}_{\ell}^{+} \rightarrow{ }^{D} \mathcal{H}_{\ell}^{-}$is the onedimensional space of functions proportional to $\chi_{\ell}^{+}$, which is the eigenfunction of the only (Proposition 4.iii) negative energy in the spectrum of $\gamma_{\mathrm{Cn}} \mathcal{H}_{\ell}^{+}$. If ${ }^{D} \mathcal{H}_{\ell}^{-}$admitted a negative energy, an eigenfunction $\kappa_{\ell}^{-}$of this energy would be sent to a negative energy eigenfunction of $\gamma_{\mathrm{ch}} \mathcal{H}_{\ell}^{+}$by the injective map $\mathcal{D}_{\ell}^{+}$; i.e., we may assume that $\mathcal{D}_{\ell}^{+} \kappa_{\ell}^{-}=\chi_{\ell}^{+}$. However, it follows from (154) that the general solution of the differential equation $\mathcal{D}_{\ell}^{+} \kappa=\chi_{\ell}^{+}$is $\tau_{\ell}^{-}+\alpha \chi_{\ell}^{-}$and, given that $\tau_{\ell}^{-}(x=0)>0$, we need $\alpha \neq 0$ for $\tau_{\ell}^{-}+\alpha \chi_{\bar{\ell}}^{-}$to equal zero at $x=0$ and, since $\alpha \neq 0$, the resulting function diverges as $x \rightarrow-\infty$ and therefore does not belong to ${ }^{D} \mathcal{H}_{\ell}^{-}$, so we reach a contradiction and conclude that ${ }^{D} \mathcal{H}_{\ell}^{-}$ has a non-negative spectrum. This allows us to prove that $\mathcal{D}_{\ell}^{-}:{ }^{\gamma_{\mathrm{Cn}}} \mathcal{H}_{\ell}^{+} \rightarrow{ }^{D} \mathcal{H}_{\ell}^{-}$is surjective proceeding as above: expand ${ }^{D} \mathcal{H}_{\ell}^{-} \ni \psi^{-}=\int d E s(E)^{D} \psi_{E}^{-}$in a basis of generalized eigenfunctions ${ }^{D} \psi_{E}^{-}$of the positive definite operator ${ }^{D} \mathcal{H}_{\ell}^{-}$. Consider the function

$$
\hat{\psi}^{-}=\int d E\left(\frac{s(E)}{w_{\ell}^{2}+E}\right) D_{\psi_{E}^{-}} .
$$

Note that $\mathcal{D}_{\ell}^{+} \hat{\psi}^{-}$belongs to the domain of ${ }^{\gamma_{\mathrm{Ch}}} \mathcal{H}_{\ell}^{+}$and that $\mathcal{D}_{\ell}^{-}$sends it to $\psi^{-}$.

\section{SUMMARY}

In what follows we enumerate the subjects addressed (items 1 and 2 below) and the results proven (items 3 and 4) in this work:

\section{A. Stability of scalar field as an indicator of gravitational stability}

The stability of a scalar test field on a given spacetime is oftentimes taken as indicative of linear gravitational stability. $\mathrm{SAdS}_{4}$ offers an example of how naive this idea can be: although there is a single choice of boundary condition for a scalar field on $\mathrm{SAdS}_{4}$, under which the field is stable, there are infinitely many possible dynamics for gravitational perturbations. If any mixture of Dirichlet, Neumann or Robin boundary conditions with $\gamma$ below the critical value is chosen for the different modes, the evolution of gravitational perturbations will be stable. If, on the contrary, a Robin boundary condition with $\gamma$ above the critical value is allowed for a single mode, the perturbation will be unstable, the instability being signaled by an exponentially growing mode similar to (138). Similar comments apply to Maxwell fields on $\mathrm{SAdS}_{4}$.

\section{B. Naturalness of Robin boundary conditions}

Robin boundary conditions on the $1+1$ auxiliary fields satisfying (15)-(16) are enforced by the self-consistence of the linear perturbation treatment in nakedly singular spacetimes (see references [29,30]). They are also a natural choice when studying gravitational perturbations of asymptotically AdS spacetimes, as they result from the imposition of Dirichlet or Neumann conditions on geometrically meaningful fields, as discussed in Sec. IV B 2. Robin conditions also arise in the context of AdS-CFT dualities, and if we want to preserve the even/odd duality in $\mathrm{SAdS}_{4}$ (see item 4 below).

\section{Robin instabilities in $\mathbf{1}+\mathbf{1}$ wave equations}

For any physical system reducing to Eq. (13) on the $x<0$ half space with a nonsingular potential, there are instabilities for high enough Robin parameter $\gamma$. If the potential is nonnegative, there is a critical value $\gamma_{c}>0$ such that the set of unstable boundary conditions is of the form $\gamma>\gamma_{c}$. A number of properties about the energy spectrum of the associated quantum Hamiltonian on a half line and its bound state are given in Secs. III A and III B (Propositions 1-6). The mechanism triggering the instabilities of (13) is explained within Sec. IIIC and illustrated using simple toy models in Sec. III D.

\section{Even/odd duality and stability of $\mathrm{SAdS}_{4}$}

Four-dimensional Schwarzschild black holes exhibit a unique feature of a duality exchanging even and odd modes, which is due to the fact that the corresponding potentials form a supersymmetric pair. This is used in [2] to extend to the even sector the proof of nonmodal stability for Schwarzschild black holes when $\Lambda \geq 0$. In the asymptotically AdS case, however, the even/odd duality is obstructed by the boundary conditions at the timelike boundary. We have found that there are only two boundary conditions compatible with the even/odd symmetry, Dirichlet in the 
even sector and Robin with a particular, $(\ell, m)$-dependent, $\gamma$ in the odd one, and vice versa, with only the first one leading to a stable dynamic (Proposition 7 in Sec. IV). An explicit unstable even gravitational mode for a specific $\gamma$ is shown in this section together with its effect on the background geometry.

\section{ACKNOWLEDGMENTS}

This work was partially funded by Grant Nos. PIP 11220080102479 (Conicet-Argentina) and 30720110101569CB (Universidad Nacional de Córdoba). B. A. is supported by a fellowship from Conicet.
[1] G. Dotti, Nonmodal Linear Stability of the Schwarzschild Black Hole, Phys. Rev. Lett. 112, 191101 (2014).

[2] G. Dotti, Black hole nonmodal linear stability: The Schwarzschild (A)dS cases, Classical Quantum Gravity 33, 205005 (2016).

[3] S. Chandrasekhar, On algebraically special perturbations of black holes, Proc. R. Soc. A 392, 1 (1984).

[4] G. Gibbons and S. A. Hartnoll, A Gravitational instability in higher dimensions, Phys. Rev. D 66, 064024 (2002).

[5] A. Ishibashi and H. Kodama, Perturbations and stability of static black holes in higher dimensions, Prog. Theor. Phys. Suppl. 189, 165 (2011).

[6] C. Garraffo and G. Giribet, The Lovelock black holes, Mod. Phys. Lett. A 23, 1801 (2008).

[7] H. Kodama and A. Ishibashi, Stability of generalized static black holes in higher dimensions, arXiv:gr-qc/0312012.

[8] G. Dotti and R. J. Gleiser, Linear stability of EinsteinGauss-Bonnet static spacetimes. Part I. Tensor perturbations, Phys. Rev. D 72, 044018 (2005).

[9] R. J. Gleiser and G. Dotti, Linear stability of EinsteinGauss-Bonnet static spacetimes. Part II: Vector and scalar perturbations, Phys. Rev. D 72, 124002 (2005).

[10] T. Takahashi and J. Soda, Master equations for gravitational perturbations of static Lovelock black holes in higher dimensions, Prog. Theor. Phys. 124, 911 (2010).

[11] A. Anabalon, D. Astefanesei, and J. Oliva, Hairy black hole stability in AdS, quantum mechanics on the half-line and holography, J. High Energy Phys. 10 (2015) 068.

[12] A. Ishibashi and R. M. Wald, Dynamics in nonglobally hyperbolic static space-times. 2. General analysis of prescriptions for dynamics, Classical Quantum Gravity 20, 3815 (2003).

[13] A. Ishibashi and R. M. Wald, Dynamics in nonglobally hyperbolic static space-times. 3. Anti-de Sitter space-time, Classical Quantum Gravity 21, 2981 (2004).

[14] G. H. Holzegel and C. M. Warnick, Boundedness and growth for the massive wave equation on asymptotically anti-de Sitter black holes, J. Funct. Anal. 266, 2436 (2014).

[15] C. M. Warnick, The Massive wave equation in asymptotically AdS spacetimes, Commun. Math. Phys. 321, 85 (2013).

[16] G. Holzegel and J. Smulevici, Decay properties of KleinGordon fields on Kerr-AdS spacetimes, Commun. Pure Appl. Math. 66, 1751 (2013).

[17] G. Holzegel, On the massive wave equation on slowly rotating Kerr-AdS spacetimes, Commun. Math. Phys. 294, 169 (2010).
[18] G. Holzegel and J. Smulevici, Quasimodes and a lower bound on the uniform energy decay rate for Kerr-AdS spacetimes, Anal. PDE 7, 1057 (2014).

[19] R. M. Wald, Dynamics in nonglobally hyperbolic, static space-times, J. Math. Phys. (N.Y.) 21, 2802 (1980).

[20] E. Chaverra, N. Ortiz, and O. Sarbach, Linear perturbations of self-gravitating spherically symmetric configurations, Phys. Rev. D 87, 044015 (2013).

[21] M. Reed and B. Simon, Fourier Analysis, Self-Adjointness, Methods of Modern Mathematical Physics Vol. 2 (Academic Press, New York, 1975).

[22] V. Cardoso and J.P. S. Lemos, Quasinormal modes of Schwarzschild anti-de Sitter black holes: Electromagnetic and gravitational perturbations, Phys. Rev. D 64, 084017 (2001).

[23] R. Wald, Note on the stability of the Schwarzschild metric, J. Math. Phys. (N.Y.) 20, 1056 (1979); Erratum, J. Math. Phys. (N.Y.) 21, 218(E) (1980).

[24] R. H. Price, Nonspherical perturbations of relativistic gravitational collapse. 1. Scalar and gravitational perturbations, Phys. Rev. D 5, 2419 (1972).

[25] P. R. Brady, C. M. Chambers, W. Krivan, and P. Laguna, Telling tails in the presence of a cosmological constant, Phys. Rev. D 55, 7538 (1997).

[26] P. J. Schmid, Nonmodal stability theory, Annu. Rev. Fluid Mech. 39, 129 (2007).

[27] I. Bakas, Energy-momentum/Cotton tensor duality for AdS (4) black holes, J. High Energy Phys. 01 (2009) 003.

[28] U. Hussain, I. Booth, and H. K. Kunduri, Master equation as a radial constraint, Phys. Rev. D 93, 123001 (2016).

[29] G. W. Gibbons, S. A. Hartnoll, and A. Ishibashi, On the stability of naked singularities, Prog. Theor. Phys. 113, 963 (2005).

[30] R. J. Gleiser and G. Dotti, Instability of the negative mass Schwarzschild naked singularity, Classical Quantum Gravity 23, 5063 (2006).

[31] G. Dotti and R. J. Gleiser, The initial value problem for linearized gravitational perturbations of the Schwarzchild naked singularity, Classical Quantum Gravity 26, 215002 (2009).

[32] B. Araneda and G. Dotti, Petrov type of linearly perturbed type D spacetimes, Classical Quantum Gravity 32, 195013 (2015).

[33] S. Chandrasekhar, The Mathematical Theory of Black Holes, 2nd ed. (Oxford University Press, New York, 1992), p. 160. 